\title{
Elasticity and dynamics of uniaxial nematic liquid crystal with defects: Nemator model
}

\author{
Natalie Aryasova $\circledast^{1,2}$ and Sergij V. Shiyanovskii $\odot^{2,3, *}$ \\ ${ }^{1}$ Physics of Crystals Department, Institute of Physics, National Academy of Sciences, Prospekt Nauki 46, 03028, Kyiv, Ukraine \\ ${ }^{2}$ Advanced Materials and Liquid Crystal Institute, Kent State University, Kent, Ohio 44242, USA \\ ${ }^{3}$ Chemical Physics Interdisciplinary Program, Kent State University, Kent, Ohio, 44242, USA
}

(Received 30 June 2020; accepted 11 November 2020; published 15 December 2020)

\begin{abstract}
We present a model of static and dynamic properties of uniaxial nematic liquid crystals that brings together the advantages of the Oseen-Frank (OF), Landau-de Gennes (LdG), and Ericksen-Leslie (EL) models. The model is based on the introduced vector $\mathbf{N}=\sqrt{s} \hat{\mathbf{n}}$, called nemator, which defines both the director $\hat{\mathbf{n}}$ and the uniaxial order parameter $s$. The equilibrium nemator field is determined from minimization of the free energy, which contains the microscopic and macroscopic parts. The proposed microscopic term combines the features of the LdG and Maier-Saupe models, and its minimization provides a good fit of temperature dependence of $s$ in the entire nematic phase. We derive the macroscopic part as a complete set of elastic, magnetic, dielectric and flexoelectric terms. The dynamic equations for the nemator, flow velocity, and pressure are derived using the conservation laws of mass, linear and angular momenta, and energy. The nemator model contains no nemator gradients and thus does not create a singularity when $\mathbf{N}$ flips, allowing simulations of static and dynamic patterns with semi-integer disclinations and other defects. Comparison of the nemator model with the OF, LdG, and EL models reveals that the material parameters and viscous coefficients in the nemator model are nonzero when $\mathbf{N} \rightarrow 0$ and can be determined from the existing experimental data.
\end{abstract}

DOI: 10.1103/PhysRevResearch.2.043373

\section{INTRODUCTION}

Liquid crystals (LCs) have a rich, more than a century long history [1], being the first and the most studied example of the orientationally ordered soft matter systems. The unique combination of LC properties, such as dielectric anisotropy, birefringence, orientational elasticity, anisotropic fluidity and sensitivity to the applied electric and magnetic fields, stimulated the development of the multibillion display and optical communications industries during the last 50 years. Recently the concepts and models of LC physics have become a basis for study of active matter, a field of physics that explores selforganization and dynamics of living and other nonequilibrium systems on length scales from nanometers to meters $[2,3]$.

The theory of static and dynamic properties of LCs is based on the translational and orientational symmetry of LC phases. The uniaxial nematic LC (NLC) is the simplest, most symmetric phase, which is anisotropic, axially symmetric, nonpolar and translationally homogeneous. The axially symmetric medium is usually described by a vector, while a proper order parameter of anisotropic nonpolar medium is a second rank tensor. This duality has caused the development of two alternative approaches for description of a uniaxial NLC.

\footnotetext{
*sshiyano@kent.edu

Published by the American Physical Society under the terms of the Creative Commons Attribution 4.0 International license. Further distribution of this work must maintain attribution to the author(s) and the published article's title, journal citation, and DOI.
}

The traditional vector approach, introduced by Oseen [4] and Frank [5], describes a uniaxial NLC as a field of the unit vector $\hat{\mathbf{n}}(\mathbf{r})$, called the director, oriented along the symmetry axis. The nonpolar nature of NLCs implies that all their properties are invariant under the $\hat{\mathbf{n}} \rightarrow-\hat{\mathbf{n}}$ transformation. The Oseen-Frank (OF) elastic energy density $f_{\mathrm{OF}}^{(\mathrm{el})}\left(n_{i}, n_{j, k}\right)$ is defined as a complete set of invariant terms which are linear and quadratic on the director spatial derivatives $n_{j, k}=\partial n_{j} / \partial x_{k}$. Each term contains the phenomenological coefficient called Frank elastic modulus. The elastic moduli are functions of the uniaxial order parameter $s$, which is assumed to remain spatially constant. The linear term exists only in chiral materials with no inversion symmetry and causes the spontaneous twist deformation, creating the chiral nematic (cholesteric) phase.

The OF elasticity model is widely used in experimental studies and computer simulations of distortions in NLCs because it has four elastic terms and the experimental methods to determine the elastic moduli are well established; however, the model also has well-known limitations. First, the assumption of constant $s$ is not valid near the cores of point and linear defects. In defects the director is undefined and the elastic energy density has a singularity. This assumption is lifted in the generalized $\mathrm{OF}$ (gOF) model proposed by Poniewierski and Sluckin [6] and Ericksen [7], who included additional elastic terms with $\nabla s$; however, the corresponding elastic moduli remain unknown. The second limitation is that the both $\mathrm{OF}$ and gOF models are not well-suited for description of semi-integer disclinations, because the director looping around the core of such disclination results in the $\hat{\mathbf{n}} \rightarrow-\hat{\mathbf{n}}$ transformation, which is allowed in a NLC, but creates infinite increase of the OF and gOF elastic energy. To overcome these difficulties Ball et al. 
studied line fields and associated with them surface discontinuities of the director field [8-10]; however, the proposed method is rather complicated, especially when configuration of disclinations is undetermined.

The Landau-de Gennes ( $\mathrm{LdG}$ ) model [11] provides an alternative description based on the traceless symmetric $\mathbf{Q}$ tensor, $Q_{i j}=s\left(\hat{n}_{i} \hat{n}_{j}-\delta_{i j} / 3\right)+p\left(\hat{l}_{i} \hat{l}_{j}-\hat{m}_{i} \hat{m}_{j}\right)$ [11,12], where $s$ and $p$ are the uniaxial and biaxial orientational order parameters, respectively, and $\hat{\mathbf{l}}, \hat{\mathbf{m}}$, and $\hat{\mathbf{n}}$ are the eigenbasis, that defines the diagonal representation of $\mathbf{Q}=$ $\operatorname{diag}(p-s / 3,-p-s / 3,2 s / 3)$. The LdG free energy density $f_{\mathrm{LdG}}=f_{\mathrm{LdG}}^{\text {(micro) }}+f_{\mathrm{LdG}}^{\text {(macro) }}$ is considered as a sum of the microscopic term $f_{\mathrm{LdG}}^{\text {(micro) }}(s, p)$, which determines the equilibrium values of $s$ and $p$ in a homogeneous system, and the macroscopic term $f_{\mathrm{LdG}}^{\text {(macro) }}\left(Q_{i j}, Q_{i j, k}\right)$ that contains the contributions of the elastic energy caused by orientational inhomogeneity and of interactions with electric and magnetic fields. The microscopic term $f_{\mathrm{LdG}}^{\text {(micro) }}(s, p)$ is derived as an invariant expansion on $\mathbf{Q}$ being a polynomial function of the invariants $I_{2}=\operatorname{Tr} \mathbf{Q}^{2}=\frac{2}{3} s^{2}+2 p^{2}$ and $I_{3}=\operatorname{Tr} \mathbf{Q}^{3}=$ $s\left(\frac{2}{9} s^{2}+2 p^{2}\right)$, and the macroscopic term $f_{\mathrm{LdG}}^{(\text {macro })}\left(Q_{i j}, Q_{i j, k}\right)$ is a sum of invariant products of the material parameters, tensor components $Q_{i j}$, their spatial derivatives $Q_{i j, k}=\partial Q_{i j} / \partial x_{k}$ and/or external fields. The fourth order expansion usually used for $f_{\mathrm{LdG}}^{\text {(micro) }}(s, p)$ is valid near nematic-isotropic phase transition, but may give nonphysical values of order parameter $s>1$ at lower temperatures. The second-order expansion for the elastic term in $f_{\mathrm{LdG}}^{\text {(macro) }}\left(Q_{i j}, Q_{i j, k}\right)$ contains three (two bulk and one divergence) elastic constants instead of four in the OF elasticity model, while in the higher order expansions the number of elastic terms drastically increases. The next non-negative expansion for the elastic energy is of the fourth order and contains 18 bulk and four surface elastic terms [13]. Using the integrity basis approach, Longa and Trebin have determined the complete set of irreducible invariants for biaxial nematics that contains 36 elastic terms and three chiral terms [14]. Unfortunately, it is impossible to determine the corresponding 39 material constants from the experiments in the uniaxial nematic phase.

Based on the OF and LdG elasticity models, respectively, the director and Q-tensor representations have been advanced to study various phenomena and effects in the nematics, such as flexoelectricity and order electricity $[15,16]$, defect structure [17-19] and dynamic properties [20-27].

Both representations work very well in their domains of applicability. The director (three variables with one condition) is an efficient tool to study static and dynamic patterns of a defect-free uniaxial NLC, where order parameter variations are negligibly small. On the other hand, the symmetric traceless Q-tensor representation is more complex (five variables), but it provides an adequate description of uniaxial and biaxial NLCs with defects.

In this paper, we present a model of static and dynamic properties of uniaxial NLCs, which combines the advantages of the director and Q-tensor models. The model is based on the vector of the variable length $\mathbf{N}$, called nemator. Nemator contains information on the amplitude $s$ and direction $\hat{\mathbf{n}}$ of the uniaxial nonpolar ordering, $\mathbf{N}=\sqrt{s} \hat{\mathbf{n}}$. The equilibrium nemator field is obtained from minimization of the free energy density $f_{\mathbf{N}}=f_{\mathbf{N}}^{(\text {micro })}(s)+f_{\mathbf{N}}^{\text {(macro) }}\left(S_{i j}, S_{k l, m}\right)$, which is a function of dyadic tensor $\mathbf{S}=\mathbf{N} \otimes \mathbf{N}$ with the components $S_{i j}=$ $N_{i} N_{j}$ and their spatial derivatives $S_{i j, k}=\partial S_{i j} / \partial x_{k}$, as shown in Sec. II. The microscopic term $f_{\mathbf{N}}^{\text {(micro) }}(s)$ depends on $\operatorname{Tr} \mathbf{S}=$ $\mathbf{N} \cdot \mathbf{N}=s$ and determines the equilibrium value of $s$ in a uniform sample in the absence of the electric and magnetic fields. We propose an expression for $f_{\mathbf{N}}^{\text {(micro) }}(s)$, which comprises the LdG-type polynomial and the term from the Maier-Saupe model with logarythmic singularity at $s \rightarrow 1$. Minimization of the proposed $f_{\mathbf{N}}^{(\text {micro) }}(s)$ provides a good one-parameter fit of $s(T)$ in the entire temperature range of the nematic phase. We derive the macroscopic part $f_{\mathbf{N}}^{\text {(macro) }}\left(S_{i j}, S_{k l, m}\right)$ as a complete set of elastic, magnetic, dielectric, and flexoelectric terms. Comparison of $f_{\mathbf{N}}^{(\text {macro })}\left(S_{i j}, S_{k l, m}\right)$ with the OF and LdG models allows us to calculate the material parameters in the nemator model from the existing experimental data. Following the continuous mechanics approach similar to Refs. [20,21], we derive the dynamic equations for the nemator $\mathbf{N}(\mathbf{r}, t)$, flow velocity $\mathbf{v}(\mathbf{r}, t)$, and pressure $p(\mathbf{r}, t)$ using the conservation laws of mass, linear and angular momenta, and energy, as shown in Sec. III. The nemator model contains no gradients $N_{i, k}$ and thus does not create a singularity when $\mathbf{N}$ flips, $\mathbf{N} \rightarrow-\mathbf{N}$, allowing simulations of static and dynamic patterns with semi-integer disclinations and other defects. All material parameters and viscous coefficients in the nemator model are nonzero when $\mathbf{N} \rightarrow 0$ and can be considered constant in the first approximation.

\section{STATIC PROPERTIES}

The equilibrium nemator field in the volume $V$ bounded by the surface $\sigma$ is found from minimization of the total free energy

$$
\Phi=\int_{V} f_{\mathbf{N}} d V+\int_{\sigma} w_{\mathbf{N}} d \sigma,
$$

where $f_{\mathbf{N}}=f_{\mathbf{N}}^{\text {(micro) }}(s)+f_{\mathbf{N}}^{\text {(macro) }}\left(S_{i j}, S_{k l, m}\right)$ and $w_{\mathbf{N}}=$ $w_{\mathbf{N}}\left(S_{i j}\right)$ is the surface anchoring term, which determines the energy density of the surface interaction with the nemator field. We consider the macroscopic term $f_{\mathbf{N}}^{\text {(macro) }}\left(S_{i j}, S_{k l, m}\right)$ as a sum of elastic $f_{\mathbf{N}}^{\text {(elast) }}\left(S_{i j}, S_{k l, m}\right)$, magnetic $f_{\mathbf{N}}^{\text {(mag) }}\left(S_{i j}\right)$, dielectric $f_{\mathbf{N}}^{(\text {diel })}\left(S_{i j}\right)$, and electrodistortion $f_{\mathbf{N}}^{(\text {ed })}\left(S_{i j}, S_{k l, m}\right)$ terms; the latter describes flexo- and order electricity. In this chapter, we describe the terms in

$$
\begin{aligned}
f_{\mathbf{N}}= & f_{\mathbf{N}}^{(\text {micro })}(s)+f_{\mathbf{N}}^{(\text {elast })}\left(S_{i j}, S_{k l, m}\right)+f_{\mathbf{N}}^{(\mathrm{mag})}\left(S_{i j}\right) \\
& +f_{\mathbf{N}}^{(\text {diel })}\left(S_{i j}\right)+f_{\mathbf{N}}^{(\text {ed })}\left(S_{i j}, S_{k l, m}\right),
\end{aligned}
$$

propose the expression for $w_{\mathbf{N}}\left(S_{i j}\right)$, and derive the equations for the equilibrium nemator field in the bulk and at the surface.

\section{A. Nemator model: The microscopic free energy}

In the nemator model, the spatial and temporal variations of the order parameter $s$ are defined by the variable length of $\mathbf{N}(\mathbf{r}, t),|\mathbf{N}(\mathbf{r}, t)|=\sqrt{s(\mathbf{r}, t)}$; thus it is important to establish a correct behavior of $f_{\mathbf{N}}^{(\text {micro })}(s)$ in the entire range $0 \leqslant s \leqslant 1$. To test the validity of $f^{\text {(micro) }}(s)$ in different models, we compare the model temperature dependence of the equilibrium scalar order parameter $s_{e q}(T)$, obtained from a solution of the 

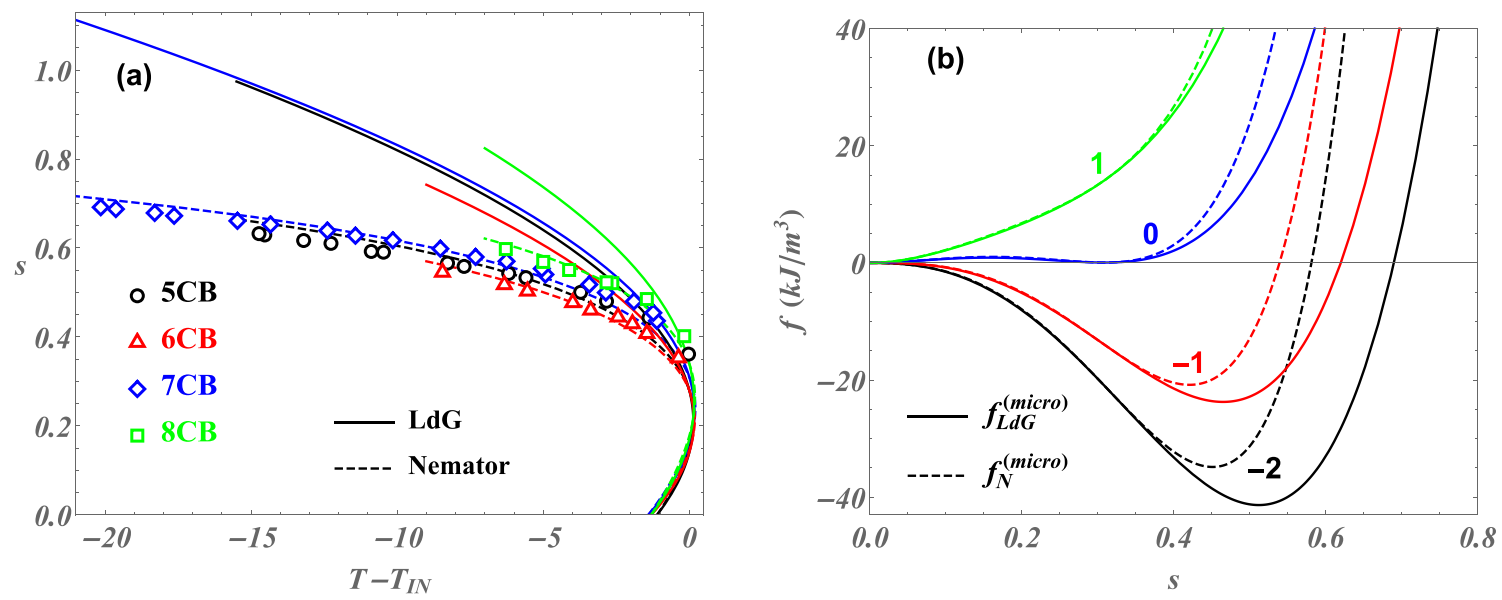

FIG. 1. Comparison of LdG and nemator models: (a) Fitting the experimental data $s(T)$ of nCB, $n=5-8$ [34], with $s_{\text {eq }}(T)$ obtained from Eq. (3) for the LdG (solid curves) and nemator (dashed curves) models; (b) $f_{\mathrm{LdG}}^{\text {(micro) }}(s)$ and $f_{\mathbf{N}}^{\text {(micro) }}(s)$ for $7 \mathrm{CB}$ at the temperatures defined by the shown values of $\tau=\left(T-T_{\mathrm{IN}}\right) /\left(T_{\mathrm{IN}}-T^{*}\right)$; all curves are obtained for parameters presented in Table I.

equation

$$
\left(\frac{\partial f^{(\text {micro })}}{\partial s}\right)_{T}=0,
$$

with the experimental results of $s(T)$ and critical behavior of the Kerr effect in the isotropic phase slightly above the temperature $T_{\mathrm{IN}}$ of the isotropic-nematic phase transition. in $s$

In the $\mathrm{LdG}$ model, $f_{\mathrm{LdG}}^{(\text {micro) }}(s)$ is a fourth-order polynomial

$$
f_{\mathrm{LdG}}^{(\text {micro })}(s)=\frac{1}{2} a^{\prime}\left(T-T^{*}\right) s^{2}-\frac{1}{3} b s^{3}+\frac{1}{4} c s^{4},
$$

where $T^{*}$ is the low-temperature limit of the isotropic phase and $a^{\prime}, b$, and $c$ are the material parameters. Equation (4) is justified for small $s$ and yields reasonable $s_{e q}$ at temperatures near $T_{\mathrm{IN}}$; however, the nonphysical values, $s_{e q}>1$, may appear at lower temperatures, Fig. 1. In the mean-field Maier-Saupe (MS) model [28], $s>1$ values are not possible, since the free energy density has the singularity $f_{M S}^{\text {(micro) }} \propto$ $-\log (1-s)$ when $s \rightarrow 1$ [28-30]. The MS model predicts for all materials the same free energy profile $f_{M S}^{(\text {micro) }}(s)$ and temperature dependence $s_{e q}(T)$; however, the predicted $s_{e q}(T)$ exhibits the significantly wider metastable regions of isotropic and nematic phases than the observed in the experiments. We propose the expression for $f_{\mathbf{N}}^{(\text {micro })}(s)$ that combines the advantages of the LdG and MS models

$$
f_{\mathbf{N}}^{(\text {micro })}(s)=\frac{1}{2} a^{\prime}\left(T-T^{*}\right) s^{2}-\frac{1}{3} b^{\prime} T s^{3}+c^{\prime} T B_{s}(4,0),
$$

where the first two terms are similar to the LdG model and the incomplete beta function $B_{s}(k, 0)=-\log (1-s)-$ $\sum_{j=1}^{k-1} s^{j} / j$, see, e.g., Ref. [31], provides the logarithmic singularity and does not interfere with the first two terms, as $B_{s}(k, 0)=s^{k} / k$ when $s \rightarrow 0$ and $\partial B_{s}(k, 0) / \partial s=$ $s^{k-1} /(1-s)$. To employ Eqs. (4) and (5) in a nematic phase with a wide temperature range, we include the $T$ factor in the coefficients of the higher terms, assuming $b=b^{\prime} T$ and $c=$ $c^{\prime} T$. The $T$ factor appears in $f^{(\text {micro) }}(s)=U-T S$ because the energy $U$ is caused by the pair intermolecular interaction and is quadratic on $s$, but the entropy $S$ also contains higher orders of $s$; similar considerations were presented in Refs. [32,33].

To test the validity of $f_{\mathrm{LdG}}^{\text {(micro) }}(s)$, Eq. (4), and $f_{\mathbf{N}}^{\text {(micro) }}(s)$, Eq. (5), we calculate $s_{\text {eq }}(T)$ from the corresponding solutions of Eq. (3)

$$
\begin{gathered}
\frac{d f_{\mathrm{LdG}}^{(\mathrm{micro})}}{d s}=a^{\prime}\left(T-T^{*}\right) s-b^{\prime} T s^{2}+c^{\prime} T s^{3}=0, \\
\frac{d f_{\mathbf{N}}^{(\mathrm{micro})}}{d s}=a^{\prime}\left(T-T^{*}\right) s-b^{\prime} T s^{2}+c^{\prime} T \frac{s^{3}}{1-s}=0,
\end{gathered}
$$

and use it to fit the experimental dependence for the 4-cyano$4^{\prime}$-n-alkylbiphenyl series ( $\left.\mathrm{nCB}\right), n=5-8$, determined from the measurements of the birefringence [34], Fig. 1. During the fitting $s_{\mathrm{eq}}(T)$ in the nematic phase, it is also important to reconstruct its hidden part $s_{\text {eq }}(T)<s_{\text {IN }}$, which corresponds to the metastable and unstable nematic states; the hidden part reveals the profile $f^{\text {(micro) }}(s)$ for small $s<s_{\mathrm{IN}}$ that controls the structure and properties of the defects' cores in the nematic phase. Thus, the parameters $a^{\prime}$ and $T_{\mathrm{IN}}-T^{*}$ in Eqs. (4)-(7) are selected from the Kerr effect data in the isotropic phase slightly above $T_{\mathrm{IN}}$ [35], Table I, and are set the same for $f_{\mathrm{LdG}}^{\text {(micro) }}(s)$ and $f_{\mathbf{N}}^{(\text {micro) }}(s)$. The condition of the phase transition $f^{\text {(micro) }}(s)=0$ at $T_{\mathrm{IN}}$ imposes a constrain between $b^{\prime}$ and $c^{\prime}$; thus for both models $s_{\mathrm{eq}}(T)$ is a function with a single fitting parameter, which we choose as the order parameter $s_{\mathrm{IN}}=s_{\text {eq }}\left(T_{\mathrm{IN}}\right)$ at the isotropic-nematic phase transition. The nemator model $s_{\text {eq }}(T)$, Eq. (5), dashed curves in Fig. 1(a),

TABLE I. Parameters of nCB, $n=5-8$, used for fitting of $s(T)$ : $T_{\mathrm{IN}}, T_{\mathrm{IN}}-T^{*}$ and $a^{\prime}$ are determined by Coles from the Kerr effect measurements [35] and $s_{\mathrm{IN}}$ is the single fitting parameter.

\begin{tabular}{lcccr}
\hline \hline & $5 \mathrm{CB}$ & $6 \mathrm{CB}$ & $7 \mathrm{CB}$ & $8 \mathrm{CB}$ \\
\hline$T_{\mathrm{IN}}(\mathrm{K})$ & 308.3 & 302.3 & 315.1 & 313.8 \\
$T_{\mathrm{IN}}-T^{*}(\mathrm{~K})$ & 1.1 & 1.3 & 1.4 & 1.3 \\
$a^{\prime}\left(\mathrm{MJ} \mathrm{m}^{-3} \mathrm{~K}^{-1}\right)$ & 0.13 & 0.15 & 0.21 & 0.18 \\
$s_{\mathrm{IN}}$, fitting value & 0.28 & 0.28 & 0.31 & 0.34 \\
\hline \hline
\end{tabular}


provides a good fit in the entire region of nematic phase with $s_{\mathrm{IN}}$, shown in Table I. On the other hand, the LdG model $s_{\text {eq }}(T)$ is much steeper than the experimental dependences for any $s_{\mathrm{IN}}$; the LdG model solid curves in Fig. 1(a) are calculated for the same values of $s_{\mathrm{IN}}$ as the dashed curves of the nemator model.

Comparison of the profiles $f_{\mathrm{LdG}}^{(\text {micro) }}(s)$, Eq. (4), and $f_{\mathbf{N}}^{(\text {micro) }}(s)$, Eq. (5), presented in Fig. 1(b) for 7CB at different temperatures, shows that $f_{\mathbf{N}}^{\text {(micro) }}(s)$ practically coincides with $f_{\mathrm{LdG}}^{\text {(micro) }}(s)$ for $s<0.4$, but reaches its minimum at $T<T_{\mathrm{IN}}$ at smaller $s=s_{\text {eq }}$ than $f_{\mathrm{LdG}}^{\text {(micro) }}(s)$, and has a substantially steeper increase for $s>s_{\text {eq }}$. The proposed $f_{\mathbf{N}}^{(\text {micro) }}(s)$ is justified for $0 \leqslant s<1$ in the entire nematic temperature range because (a) it exhibits the logarithmic singularity when $s \rightarrow 1$; (b) $f_{\mathbf{N}}^{\text {(micro) }}(s)$ describes adequately the thermodynamics near the isotropic-nematic phase transition since its parameters match the experimental values of $T_{\mathrm{IN}}, T_{\mathrm{IN}}-T^{*}$, and $a^{\prime}$, determined from the Kerr effect measurements in the isotropic phase, Table I; and (c) the temperature dependence of $s_{\text {eq }}(T)$, which defines the position of $f_{\mathbf{N}}^{\text {(micro) }}(s)$ minimum, fits the experimental data in the entire nematic phase. Thus, $f_{\mathbf{N}}^{(\text {micro })}(s)$ is used in the nemator model to control the length of $\mathbf{N}(\mathbf{r}, t)$, $|\mathbf{N}(\mathbf{r}, t)|=\sqrt{s(\mathbf{r}, t)}$.

\section{B. Nemator model: The elastic free energy}

We start with a general invariant expression

$$
f_{\mathbf{N}}^{(\text {elast })}=f_{\mathbf{N}}^{(b)}+f_{\mathbf{N}}^{(d)}+f_{\mathbf{N}}^{(c)},
$$

where the bulk term $f_{\mathbf{N}}^{(b)}=B_{i j k l m n}(\mathbf{S}) S_{i j, k} S_{l m, n}$ is a quadratic form of spatial derivatives, $f_{\mathbf{N}}^{(d)}=\left[D_{i j k l}(\mathbf{S}) S_{i j, k}\right]_{, l}$ is the divergence term, and $f_{\mathbf{N}}^{(c)}=C_{i j k l m n}(\mathbf{S}) \varepsilon_{i j k} S_{l m, n}$ is the linear on spatial derivatives chiral term. Here the tensors $B_{i j k l m n}(\mathbf{S})$, $D_{i j k l}(\mathbf{S})$, and $C_{i j k l m n}(\mathbf{S})$ are sums of products of $S_{i j}$, the Kronecker deltas $\delta_{i j}$, and the elastic moduli $M_{\alpha}(s)$, which are functions of $\operatorname{Tr} \mathbf{S}=s$. The proposed approach prohibits the bulk and divergence terms that contain second-order spatial derivatives, like $K_{13}$, the splay-bend term in the director representation; thus, the elastic free energy is bounded from below and eliminates the possibility of the Barbero-Oldano paradox behavior [36,37].

To obtain all relevant products in $f_{\mathbf{N}}^{(b)}$, we use the diagrams with the following notation: $S_{i j, k} \Leftrightarrow \overbrace{j}^{\rightarrow k}, S_{i j} \Leftrightarrow{ }^{i}-\infty^{i}, \delta_{i j} \Leftrightarrow$ i.....i, and connections correspond to the summation over proper indices. The products in $B_{i j k l m n}(\mathbf{S})$ with summations over other indices do not yield new products, because $\delta_{i p} \delta_{p k}=\delta_{i k}$, $S_{i p} \delta_{p k}=S_{i k}$, and $S_{i p} S_{p k}=s S_{i k}$. Thus, all irreducible products correspond to the diagrams presented in Fig. 2, e.g., diagram $\mathbf{2 , 1}$ corresponds to $f_{2,1}=S_{i j, k} S_{i j, k}$.

Not all terms shown in Fig. 2 are independent because some of them can be represented as linear combinations of other terms. We form the independent basis $\Gamma=$ $\left\{f_{2,1}, f_{2,2}, f_{2,3}, f_{2,4}, f_{2,5}, f_{3,2 b}, f_{3,4 b}, f_{3,5 a}\right\}$, which contains all second-order terms, $f_{2,1}=S_{i j, k} S_{i j, k}, f_{2,2}=S_{i j, j} S_{i k, k}=$ $\boldsymbol{\mu}^{2}, \quad f_{2,3}=S_{i j, k} S_{i k, j}, \quad f_{2,4}=S_{i j, j} S_{k k, i}=\boldsymbol{\mu} \cdot \nabla s$, and $f_{2,5}=$ $S_{i i, k} S_{j j, k}=(\nabla s)^{2}$, and three third-order terms that are similar to the second-order terms: $f_{3,2 b}=\boldsymbol{\mu} \cdot \mathbf{S} \cdot \boldsymbol{\mu}, f_{3,4 b}=\boldsymbol{\mu} \cdot \mathbf{S}$. $\nabla s$ and $f_{3,5 a}=\nabla s \cdot \mathbf{S} \cdot \nabla s$; here $\boldsymbol{\mu}=\nabla \cdot \mathbf{S}$. All other terms,

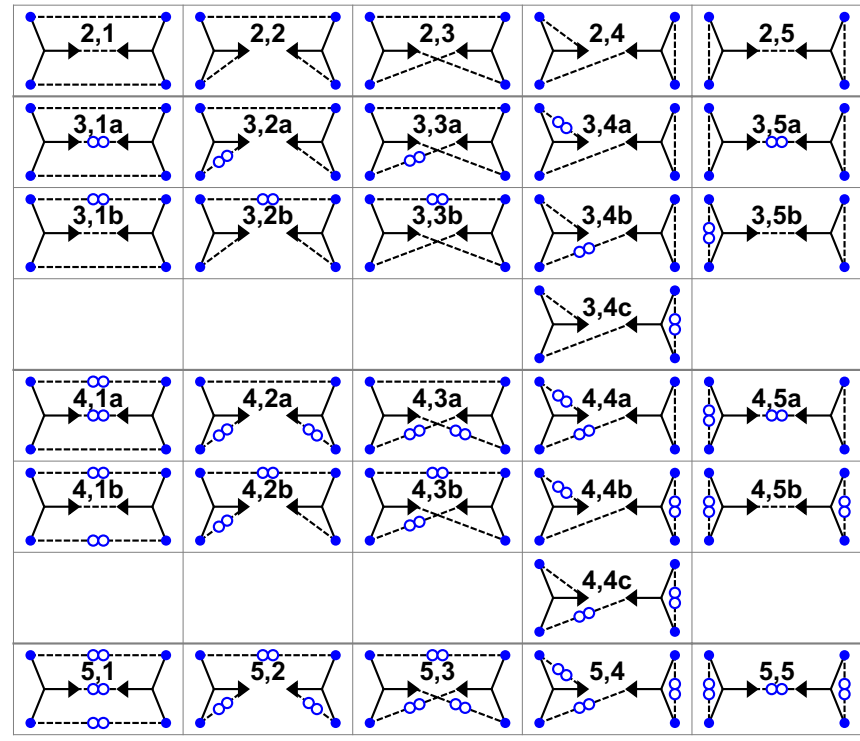

FIG. 2. The diagrams correspond to the products $f_{\alpha, \beta}$ in $f_{\mathbf{N}}^{(b)}$, Eq. (8), where $\alpha$ indicates the order on tensor $\mathbf{S}=\mathbf{N} \otimes \mathbf{N}$, and $\beta$ labels the diagram's structure. The diagram notation is $S_{i j, k} \Leftrightarrow \gamma_{j}^{\infty} \rightarrow$, $S_{i j} \Leftrightarrow{ }^{i}-\infty{ }^{i}, \delta_{i j} \Leftrightarrow i \ldots . . .$.

shown in Fig. 2, can be represented as their linear combination,

$$
f_{\alpha, \beta}=\sum_{n}\left\langle\alpha, \beta \mid \alpha_{n}, \beta_{n}\right\rangle s^{\Delta \alpha} f_{\alpha_{n}, \beta_{n}}
$$

where $f_{\alpha_{n}, \beta_{n}}=\Gamma_{n}$ is the $n^{\text {th }}$ term of the basis $\Gamma, \Delta \alpha=\alpha-$ $\alpha_{n}$, and $\left\langle\alpha, \beta \mid \alpha_{n}, \beta_{n}\right\rangle$ is the numerical expansion coefficient, shown in Table II. Note that $f_{4,1 a}=f_{4,2 a}=f_{4,3 a}, f_{4,4 a}=$ $f_{4,5 a}, f_{4,1 b}=f_{4,5 b}, f_{4,2 b}=f_{4,4 c}, f_{4,3 b}=f_{4,4 b}$, and all the fifthorder terms give the same contribution $f_{5,1}=f_{5,2}=f_{5,3}=$ $f_{5,4}=f_{5,5}$ because $S_{j l} S_{k m}=S_{j k} S_{l m}$.

The divergence term $f_{\mathbf{N}}^{(d)}$ can also be expressed through the chosen basis and it is instrumental to find its explicit form. Because $f_{\mathbf{N}}^{(d)}$ has no second-order spatial derivatives, $D_{i j k l}(\mathbf{S})$ is antisymmetric on indices $k$ and $l$, and is represented as $D_{i j k l}(\mathbf{S})=\tilde{D}_{i j k l}(\mathbf{S})-\tilde{D}_{i j l k}(\mathbf{S})$ with corresponding elastic modulus $M_{d}(s)$, where $\tilde{D}_{i j k l}(\mathbf{S})=M_{d}(s) \delta_{i k} S_{j l}$ is the only nonsymmetric tensor product. Thus, $f_{\mathbf{N}}^{(d)}$ is a difference of diagrams shown in Fig. 3(a), and reads

$$
f_{\mathbf{N}}^{(d)}=\nabla\left[M_{d}(s) \eta\right]=M_{d}(s) \nabla \boldsymbol{\eta}+M_{d}^{\prime}(s) \nabla s \cdot \boldsymbol{\eta},
$$

where $M_{d}^{\prime}(s)=d\left(M_{d}(s)\right) / d s$ and introduced in Eq. (12) vector $\boldsymbol{\eta}$ has components

$$
\eta_{i}=S_{j k} S_{i j, k}-S_{i j} S_{j k, k}=s \mu_{i}+S_{i k} s_{, k}-2 S_{i j} \mu_{j} .
$$
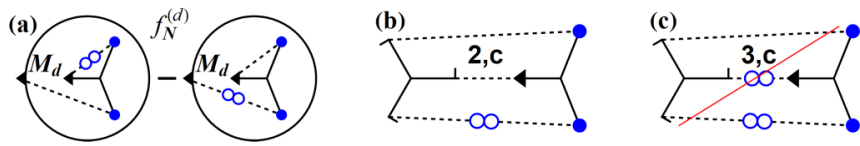

FIG. 3. The diagrams for the divergence term $f_{\mathbf{N}}^{(d)}$ (a) and for the chiral products $f_{2, c}$ (b) and $f_{3, c} \equiv 0$ (c); the diagram notation is the same as in Fig. 2 with $\partial / \partial x_{l} \Leftrightarrow D$ and $\varepsilon_{i j k} \Leftrightarrow{ }_{j}^{i} \sim^{k}$. 
TABLE II. Numerical expansion coefficients $\left\langle\alpha, \beta \mid \alpha_{n}, \beta_{n}\right\rangle$ in Eq. (9), bold, and $\underline{\left\langle\alpha, \beta \mid \alpha_{n}, \beta_{n}\right\rangle}$ in Eq. (18), underlined in parentheses.

\begin{tabular}{|c|c|c|c|c|c|c|c|c|}
\hline & 2,1 & 2,2 & 2,3 & 2,4 & 2,5 & $3,2 b$ & $3,4 b$ & $3,5 \mathrm{a}$ \\
\hline 2,1 & $\mathbf{1}(\underline{1})$ & & & & $(-1 / 3)$ & & & \\
\hline 2,2 & & $\mathbf{1}(\underline{1})$ & & $(-2 / 3)$ & $(\underline{1 / 9)}$ & & & \\
\hline 2,3 & & & $\mathbf{1}(\underline{1})$ & $(\overline{-2 / 3})$ & $(\overline{1 / 9})$ & & & \\
\hline 2,4 & & & & 1 & & & & \\
\hline 2,5 & & & & & 1 & & & \\
\hline $3,1 \mathrm{a}$ & $(-1 / 3)$ & $2(\underline{2})$ & & & $(\underline{1 / 9})$ & $-2(\underline{-2})$ & & $\mathbf{1}(\underline{2 / 3})$ \\
\hline $3,1 \mathrm{~b}$ & $\mathbf{1 / 2 ( \underline { 1 / 6 } )}$ & & & & $\mathbf{1 / 2} \overline{(1 / 18)}$ & & & \\
\hline $3,2 \mathrm{a}$ & & $\mathbf{1}(\underline{2 / 3})$ & & $(\underline{-1 / 9)}$ & $(-\overline{1 / 27})$ & $-\mathbf{1}(\underline{-1})$ & $\mathbf{1}(\underline{1})$ & $(-2 / 9)$ \\
\hline $3,2 b$ & & $(-\overline{-1 / 3})$ & & $\overline{(2 / 9)}$ & $(\overline{-1 / 27})$ & $1(\overline{1})$ & $(-\overline{2 / 3})$ & $(\underline{1 / 9)}$ \\
\hline $3,3 \mathrm{a}$ & & $\overline{\mathbf{1}(\underline{1})}$ & $(\underline{-1 / 3})$ & $1(\overline{8 / 9})$ & $(\overline{-1 / 27})$ & $-1(-1)$ & $-\overline{\mathbf{1}(-1)}$ & $\mathbf{1 ( \overline { 7 / 9 } )}$ \\
\hline $3,3 b$ & & $1(-1)$ & $1(\underline{2 / 3})$ & $(-\overline{-4 / 9})$ & $(\overline{-1 / 27})$ & $\mathbf{1}(\underline{1)}$ & $(\underline{2 / 3)}$ & $(-\overline{5 / 9})$ \\
\hline $3,4 \mathrm{a}$ & & & & 1 & & & $\overline{-1}$ & 1 \\
\hline $3,4 b$ & & & & & & & 1 & \\
\hline $3,4 \mathrm{c}$ & & & & $\mathbf{1}(\underline{2 / 3})$ & $(-2 / 9)$ & & & \\
\hline $3,5 \mathrm{a}$ & & & & & & & & 1 \\
\hline $3,5 b$ & & & & & 1 & & & \\
\hline $4,1 \mathrm{a}$ & $(-1 / 18)$ & $\mathbf{1}(\underline{1 / 3})$ & & & $(-1 / 54)$ & $\mathbf{- 1}(-1 / 3)$ & & $\mathbf{1}(\underline{2 / 9})$ \\
\hline $4,1 \mathrm{~b}$ & $\overline{(-2 / 9)}$ & & & & $\overline{1(4 / 9)}$ & & & \\
\hline $4,2 \mathrm{a}$ & & $\mathbf{1}(\underline{4 / 9})$ & & $(\underline{4 / 27})$ & $(\overline{1 / 81})$ & $\mathbf{- 1}(\underline{-1 / 3})$ & $(-2 / 3)$ & $\mathbf{1}(\underline{16 / 27})$ \\
\hline $4,2 b$ & & $(-\overline{-2 / 9})$ & & $(\overline{1 / 27})$ & $(\overline{1 / 81})$ & & $1(\underline{5 / 9})$ & $(-5 / 27)$ \\
\hline $4,3 \mathrm{a}$ & & $\mathbf{1}(1 / 3)$ & $(\underline{1 / 9})$ & $(-14 / 27)$ & $(\overline{1 / 81})$ & $\mathbf{- 1}(\underline{-1 / 3})$ & $(\overline{2 / 3)}$ & $1(-2 / 27)$ \\
\hline $4,3 \mathrm{~b}$ & & & $(-2 / 9)$ & $\mathbf{1}(16 / 27)$ & $(\overline{1 / 81})$ & & $-1(-5 / 9)$ & $\mathbf{1}(10 / 27)$ \\
\hline $4,4 \mathrm{a}$ & & & & & & & & 1 \\
\hline $4,4 \mathrm{~b}$ & & & & $\mathbf{1}(\underline{4 / 9})$ & $(\underline{2 / 27})$ & & $-1(\underline{-2 / 3)}$ & $\mathbf{1}(\underline{4 / 9})$ \\
\hline $4,4 \mathrm{c}$ & & & & $(\overline{-2 / 9})$ & $(\overline{2 / 27})$ & & $\mathbf{1}(\underline{2 / 3})$ & $(-\overline{-2 / 9})$ \\
\hline $4,5 \mathrm{a}$ & & & & & & & & 1 \\
\hline $4,5 b$ & & & & & $\mathbf{1}(\underline{4 / 9})$ & & & \\
\hline 5,1 & $(\underline{2 / 27})$ & $(-4 / 9)$ & & & $(-\overline{4 / 27})$ & $(\underline{4 / 9})$ & & $\mathbf{1}(\underline{2 / 9})$ \\
\hline 5,2 & & $(\overline{-4 / 27})$ & & $(-4 / 81)$ & $(\overline{-1 / 243})$ & $(\overline{2 / 9})$ & $(-8 / 27)$ & $\mathbf{1}(\underline{11 / 27})$ \\
\hline 5,3 & & $\overline{(-2 / 9)}$ & $(\underline{2 / 27)}$ & $(\overline{-28 / 81})$ & $(\overline{-1 / 243})$ & $(\overline{2 / 9})$ & $\overline{(8 / 27)}$ & $\overline{\mathbf{1}(\underline{1 / 9)}}$ \\
\hline 5,4 & & & & $\overline{(-4 / 27)}$ & $\overline{(-2 / 81)}$ & & & $\mathbf{1}(\overline{10 / 27})$ \\
\hline 5,5 & & & & & $(\overline{-4 / 27})$ & & & $\overline{\mathbf{1}(\underline{4 / 9)})}$ \\
\hline
\end{tabular}

Using Eqs. (10), (11), we exclude $f_{2,3}$ term from the basis and substitute it with the divergence term $f_{\mathbf{N}}^{(d)}$, because $\nabla \eta=$ $f_{2,3}-f_{2,2}$ and $\nabla s \cdot \boldsymbol{\eta}=s f_{2,4}+f_{3,5 a}-2 f_{3,4 b}$.

The chiral NLC term $f_{\mathbf{N}}^{(c)}$ contains the only independent second-order product $f_{2, c}=\varepsilon_{i j k} S_{l j} S_{i l, k}$, Fig. 3(b), where the antisymmetric Levi-Civita tensor $\varepsilon_{i j k}$ is represented in diagrams as a triskelion; the third order product, Fig. 3(c), is crossed with a red line, because it vanishes over summation $f_{3, c}=\varepsilon_{i j k} S_{l j} S_{k m} S_{i l, m}=\varepsilon_{i j k} S_{k j} S_{l m} S_{i l, m} \equiv 0$.

Combining the derived expressions for $f_{\mathbf{N}}^{(b)}, f_{\mathbf{N}}^{(d)}$, and $f_{\mathbf{N}}^{(c)}$ we result in $f_{\mathbf{N}}^{\text {(elast) }}$

$$
f_{\mathbf{N}}^{(\text {elast })}=\frac{1}{2}\left[\begin{array}{c}
M_{1} S_{i j, k} S_{i j, k}+M_{2} \boldsymbol{\mu}^{2}+M_{3} \boldsymbol{\mu} \cdot \mathbf{S} \cdot \boldsymbol{\mu}+M_{4} \boldsymbol{\mu} \cdot \nabla s \\
+M_{5} \boldsymbol{\mu} \cdot \mathbf{S} \cdot \nabla s+M_{6}(\nabla s)^{2}+M_{7} \nabla s \cdot \mathbf{S} \cdot \nabla s
\end{array}\right]+M_{c} \varepsilon_{i j k} S_{i l, k} S_{l j}+\nabla \cdot\left(M_{d} \boldsymbol{\eta}\right),
$$

where $\boldsymbol{\eta}=s \boldsymbol{\mu}+\mathbf{S} \cdot \nabla s-2 \mathbf{S} \cdot \boldsymbol{\mu}, \boldsymbol{\mu}$ is the vector with components $\mu_{i}=S_{i j, j}$, and the elastic moduli $M_{\alpha}=M_{\alpha}(s)$ are polynomial functions of $s=\mathbf{N} \cdot \mathbf{N}$. The linear on the gradient term with $M_{c}$ appears in chiral NLCs. The last term is a divergence and its volume integral is reduced to the surface integral. To determine the elastic moduli $M_{\alpha}(s)$, we compare $f_{\mathbf{N}}^{(\text {elast })}$ with the OF, $\mathrm{gOF}$, and LdG models.

Comparison with the OF and gOF models. The bounded from below OF elastic energy density $f_{\mathrm{OF}}$ is defined in terms of the director gradient

$$
f_{\mathrm{OF}}^{(\text {elast })}=\frac{1}{2} K_{1}(\operatorname{div} \hat{\mathbf{n}})^{2}+\frac{1}{2} K_{2}(\hat{\mathbf{n}} \cdot \operatorname{curl} \hat{\mathbf{n}})^{2}+K_{\mathrm{c}}(\hat{\mathbf{n}} \cdot \operatorname{curl} \hat{\mathbf{n}})+\frac{1}{2} K_{3}(\hat{\mathbf{n}} \times \operatorname{curl} \hat{\mathbf{n}})^{2}-K_{24} \operatorname{div}(\hat{\mathbf{n}} \operatorname{div} \hat{\mathbf{n}}+\hat{\mathbf{n}} \times \operatorname{curl} \hat{\mathbf{n}}),
$$

where the elastic moduli $K_{1}, K_{2}, K_{3}$, and $K_{24}$ are related to the splay, twist, bend and saddle-splay deformations, respectively. The linear term with $K_{\mathrm{c}}$ exists in the chiral nematic. The elastic moduli are considered to be functions of $s$, which is assumed to remain spatially constant. Under this assumption the last term is a divergence and is reduced to the surface integral. 
The elastic energy in the gOF model contains additional terms with the order parameter gradient $\nabla s[6,7]$

$$
\begin{aligned}
f_{\mathrm{gOF}}^{(\text {elast })}= & f_{\mathrm{OF}}^{\text {(elast) }}+\frac{1}{2} K_{5}(\nabla s)^{2}+\frac{1}{2} K_{6}(\hat{\mathbf{n}} \cdot \nabla s)^{2} \\
& +\frac{1}{2} K_{7}(\hat{\mathbf{n}} \cdot \nabla s) \operatorname{div} \hat{\mathbf{n}}+\frac{1}{2} K_{8} \nabla s \cdot[\nabla \cdot(\hat{\mathbf{n}} \otimes \hat{\mathbf{n}})] .
\end{aligned}
$$

Using the definition of the nemator $\mathbf{N}=\sqrt{s} \hat{\mathbf{n}}$, and comparing Eq. (12) with Eqs. (13) and (14), we obtain the following relations between the elastic moduli $K_{i}(s)$ and $M_{j}(s)$ :

$$
\begin{aligned}
& K_{c}=s^{2} M_{c} \\
& K_{1}=s^{2}\left(2 M_{1}+M_{2}+s M_{3}\right), \quad K_{2}=2 s^{2} M_{1} \\
& K_{3}=s^{2}\left(2 M_{1}+M_{2}\right), \quad K_{24}=s^{2}\left(M_{1}+M_{d}\right) \\
& K_{5}=M_{1}+M_{6}, \quad K_{6}=M_{2}+s M_{3}+M_{4}+s M_{5}+s M_{7}, \\
& K_{7}=s\left(2 M_{2}+2 s M_{3}-8 M_{d}+s M_{5}-2 s M_{d}^{\prime}\right) \\
& K_{8}=s\left(4 M_{d}+M_{4}+s M_{d}^{\prime}\right) .
\end{aligned}
$$

Equations (16) show that the Frank moduli $K_{1-3}(s)$ and $K_{24}(s)$ are expressed with the four nemator moduli $M_{1-3}(s)$ and $M_{d}(s)$ and vice versa. Similarly, Eqs. (17) exhibits the relations for the moduli $K_{5-8}(s)$. Note that contrary to Eqs. (13) and (14), Eq. (12) does not create a singularity when $\mathbf{N}$ flips, $\mathbf{N} \rightarrow-\mathbf{N}$, and are therefore well-suited for simulation of patterns with semi-integer disclinations.

Comparison with the LdG model. To compare orientational elasticity in the nemator and $\mathrm{LdG}$ models we represent the elastic term in the nemator model $f_{\mathbf{N}}^{(\text {(elast) }}$ in terms of the traceless uniaxial tensor $\underline{\mathbf{Q}}=\mathbf{S}-\frac{1}{3} s \mathbf{I}$, where $\mathbf{I}$ is the unit tensor. To determine $f_{\mathbf{N}}^{\text {(elast) }}=f_{\mathbf{N}}^{(b)}+f_{\mathbf{N}}^{(d)}+f_{\mathbf{N}}^{(c)}$ with the bulk $f_{\mathbf{N}}^{(b)}=$ $\underline{B}_{i j k l m n}(\underline{\mathbf{Q}}) \underline{Q}_{i j, k} \underline{Q}_{l m, n}$, divergence $\underline{f}_{\mathbf{N}}^{(d)}=\left[\underline{D}_{i j k l}(\underline{\mathbf{Q}}) \underline{Q}_{i j, k}\right]_{, l}$, and chiral $\underline{f}_{\mathbf{N}}^{(c)}=\underline{C}_{i j k l m n}(\underline{\mathbf{Q}}) \varepsilon_{i j k} \underline{Q}_{l m, n}$ terms, we use the same diagrams, Figs. 2 and 3, with substitution $S_{i j} \rightarrow \underline{Q}_{i j}$; e.g., diagram 2,1 in Fig. 2 yields the term $\underline{f}_{2,1}=\underline{Q}_{i j, k} \underline{Q}_{i j, k}$. We then expand $\underline{f}_{\alpha, \beta}$ using the same basis $\Gamma$

$$
\underline{f}_{\alpha, \beta}=\sum_{n} \underline{\left\langle\alpha, \beta \mid \alpha_{n}, \beta_{n}\right\rangle} s^{\Delta \alpha} f_{\alpha_{n}, \beta_{n}},
$$

where $\left\langle\alpha, \beta \mid \alpha_{n}, \beta_{n}\right\rangle$ are the numerical expansion coefficients, shown in Table II. Expansion (18) and relations between the chiral $\underline{f}_{2, c}=\varepsilon_{i j k} \underline{Q}_{l j} \underline{Q}_{i l, k}=f_{2, c}, \quad \underline{f}_{3, c}=\varepsilon_{i j k} \underline{Q}_{l j} \underline{Q}_{k m} \underline{Q}_{i l, m}=$ $s f_{2, c} / 3$, and divergence $\underline{f}_{\mathbf{N}}^{(d)}=f_{\mathbf{N}}^{(d)}$ terms demonstrate that $f_{\mathbf{N}}^{(\mathrm{el})}$ is also described by Eq. (12).

Note that $f_{\mathbf{N}}^{(\text {elast })}$ and $\underline{f}_{\mathbf{N}}^{(\text {elast })}$ are not equivalent. Since $\underline{f}_{2,4} \equiv$ $\underline{f}_{2,5} \equiv 0$ and $\underline{f}_{\mathbf{N}}^{\text {(elast) }}$ contains only three independent secondorder terms, the equivalence of $f_{\mathbf{N}}^{\text {(elast) }}$ and $f_{\mathbf{N}}^{\text {(elast) }}$ requires that the second-order elastic moduli $M_{\alpha}(0)$ in Eq. (12) for $f_{\mathbf{N}}^{\text {(elast) }}$ should obey the relations

$M_{5}(0)=-2 M_{2}(0) / 3, \quad M_{7}(0)=\left(M_{2}(0)-3 M_{1}(0)\right) / 9$.

Similarly, the six independent third-order terms in $f_{\mathbf{N}}^{(\mathrm{el})}$ result in the additional relations for $M_{\alpha}(0)$ in $f_{\mathbf{N}}^{(\text {elast })}$

$$
\begin{aligned}
& M_{8}(0)=M_{5}^{\prime}(0)-M_{1}^{\prime}(0)+3 M_{7}^{\prime}(0), \\
& M_{6}(0)=\left(M^{\prime}{ }_{2}(0)-M_{3}(0)-6 M_{8}(0)\right) / 3 .
\end{aligned}
$$

In our opinion, the validity of $\underline{f}_{\mathbf{N}}^{\text {(elast) }}$ and conditions (19) and (20) in Eq. (12) for $f_{\mathbf{N}}^{\text {(elast) }}$, depends on the specifics of the nematic phase. Conditions (19) and (20) are probably justified for standard nematics, e.g., 5CB, but may be invalid in materials with additional intrinsic parameters, like lyotropic chromonic liquid crystals where order parameter changes with concentrations of the components and length of aggregates [38-41].

\section{Nemator model: Interaction with external fields}

The interaction with external electric $\mathbf{E}$ and magnetic B fields can be derived from comparison with the $\mathrm{OF}$ model. Because magnetic intermolecular interaction are small, the magnetic susceptibility tensor $\chi=\chi_{\perp} \mathrm{I}+\left(\chi_{\|}-\right.$ $\left.\chi_{\perp}\right)(\hat{\mathbf{n}} \otimes \hat{\mathbf{n}})=N_{\text {mol }}\langle\boldsymbol{\kappa}\rangle$ is expressed by the components parallel $\chi_{\|}$and perpendicular $\chi_{\perp}$ to the director, and is a product of the number of molecules per unit volume $N_{\text {mol }}$ and an orientational average $\langle\boldsymbol{\kappa}\rangle$ of the molecular magnetic tensor $\kappa$. Thus $\chi_{\|}+2 \chi_{\perp}=N_{\text {mol }} \operatorname{Tr} \kappa$ is constant and the magnetic anisotropy $\chi_{a}=\chi_{\|}-\chi_{\perp}=\hat{\chi}_{a} s$ is proportional to $s$ with $\hat{\chi}_{a}=N_{\text {mol }} \kappa_{a}$ being a constant factor, and the magnetic term of the free energy in the OF model $f_{\mathrm{OF}}^{(m)}=-\frac{\chi_{a}}{2 \mu_{0}}(\hat{\mathbf{n}} \cdot \mathbf{B})^{2}$, where $\mu_{0}$ is the magnetic constant, transforms into $f_{\mathbf{N}}^{(m)}=$ $-\frac{\hat{\chi}_{a}}{2 \mu_{0}}\left[(\mathbf{N} \cdot \mathbf{B})^{2}-\frac{s}{3} B^{2}\right]$ in the nemator model.

The dielectric term of the free energy $f_{\mathrm{OF}}^{(\mathrm{diel})}=$ $-\frac{\varepsilon_{0}}{2}(\mathbf{E} \cdot \boldsymbol{\varepsilon} \cdot \mathbf{E})$ is more complex than the magnetic one, because the electric intermolecular interactions are strong and the dielectric permittivity tensor $\boldsymbol{\varepsilon}=\varepsilon_{\perp} \mathrm{I}+\left(\varepsilon_{\|}-\varepsilon_{\perp}\right)(\hat{\mathbf{n}} \otimes \hat{\mathbf{n}})$ is the nonlinear function of $s$; here $\varepsilon_{\|}$and $\varepsilon_{\perp}$ are components of the tensor parallel and perpendicular to the director, respectively. Still the dielectric anisotropy $\varepsilon_{a}=\varepsilon_{\|}-\varepsilon_{\perp} \rightarrow 0$ when $s \rightarrow 0$ and can be represented as $\varepsilon_{a}(s)=\hat{\varepsilon}_{a}(s) s$ resulting in $\boldsymbol{\varepsilon}=\varepsilon_{\perp}(s) \mathrm{I}+\hat{\varepsilon}_{a}(s) \mathbf{S}$ for the nemator model.

In addition to the dielectric term the bulk free energy associated with the external electric field also contains the electrodistortion term $f^{(\text {ed })}=-\mathbf{E} \cdot \mathbf{P}^{(\text {ed })}$, where the polarization $\mathbf{P}^{(e d)}$ is caused by the distortion of the orientational ordering.

To obtain all possible terms in $\mathbf{P}_{\mathbf{N}}^{(\text {ed })}$, we build the linear on $S_{i j, k}$ vector diagrams, Fig. 4 , using the same notation as in Fig. 2, e.g., diagrams $\mathbf{1 , 1}$ and $\mathbf{2 , 1 a}$ correspond to $\mathbf{p}_{1,1}=$ $\nabla \cdot \mathbf{S}=\boldsymbol{\mu}$ and $\mathbf{p}_{2,1 a}=\mathbf{S} \cdot \boldsymbol{\mu}$, respectively.

Keeping the similarity with representation (8) of $f_{\mathbf{N}}^{(\mathrm{el})}$, we express $\mathbf{P}_{\mathbf{N}}^{(\text {ed) }}$ through the basis of independent vector terms $\Gamma^{(\mathbf{p})}=\left\{\mathbf{p}_{1,1}, \quad \mathbf{p}_{1,2}, \mathbf{p}_{2,1 a}, \mathbf{p}_{2,2 a}\right\}=\{\boldsymbol{\mu}, \nabla s, \mathbf{S} \cdot \boldsymbol{\mu}, \mathbf{S} \cdot \nabla s\}$,

$$
\mathbf{P}_{\mathbf{N}}^{(\mathrm{ed})}=\xi_{1}(s) \boldsymbol{\mu}+\xi_{2}(s) \nabla s+\xi_{3}(s) \mathbf{S} \cdot \boldsymbol{\mu}+\xi_{4}(s) \mathbf{S} \cdot \nabla s,
$$

where $\xi_{1}(s)$ are the materials parameters, which we call electrodistortion coefficients.

Similarly, any term, shown in Fig. 4, can be represented as

$$
\mathbf{p}_{\alpha, \beta}=\sum_{n}\left\langle\alpha, \beta \mid \alpha_{n}, \beta_{n}\right\rangle_{(\mathbf{p})}{ }^{s^{\Delta \alpha}} \mathbf{p}_{\alpha_{n}, \beta_{n}},
$$

where $\mathbf{p}_{\alpha_{n}, \beta_{n}}=\Gamma_{n}^{(\mathbf{p})}$ is the $n^{\text {th }}$ term of the basis $\Gamma, \Delta \alpha=\alpha-$ $\alpha_{n}$, and $\left\langle\alpha, \beta \mid \alpha_{n}, \beta_{n}\right\rangle_{(\mathbf{p})}$ is the numerical expansion coefficient, shown in Table III. 


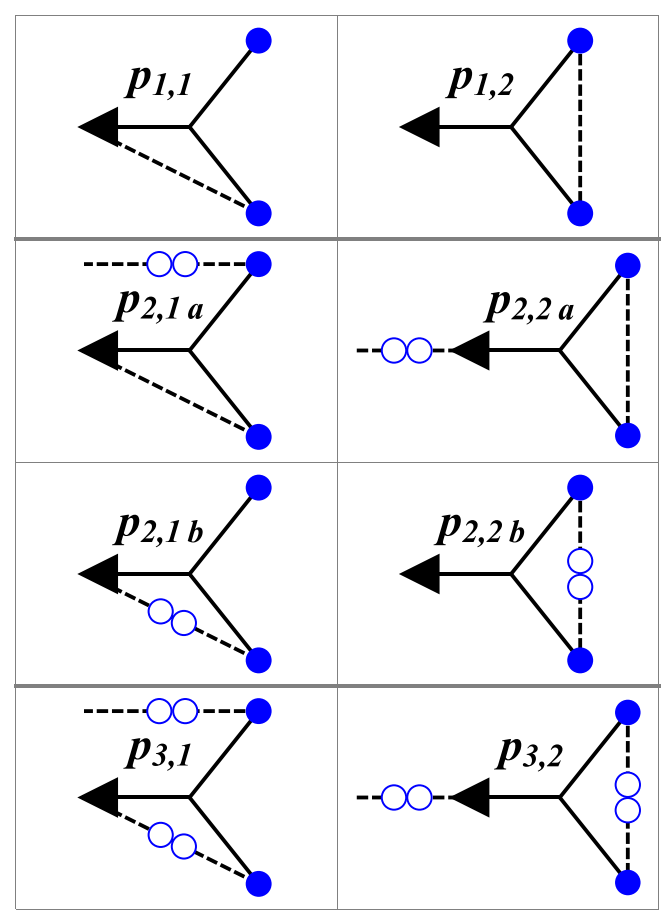

FIG. 4. The diagrams for the electrodistortion term $f_{\mathbf{N}}^{(\text {ed) }}$; the diagram notation is the same as in Fig. 2.

In the director model, $\mathbf{P}_{\mathrm{OF}}^{(\mathrm{ed})}=\mathbf{P}_{\mathrm{OF}}^{(f l)}+\mathbf{P}_{\mathrm{OF}}^{(o)}$ splits on the flexopolarization $\mathbf{P}_{\mathrm{OF}}^{(f l)}=e_{1} \hat{\mathbf{n}} \operatorname{div} \hat{\mathbf{n}}-e_{3} \hat{\mathbf{n}} \times$ curl$\hat{\mathbf{n}}$, produced by the director distortions [15], and the order polarization $\mathbf{P}_{\mathrm{OF}}^{(o)}=r_{1}(\hat{\mathbf{n}} \cdot \nabla s) \hat{\mathbf{n}}+r_{2} \nabla s$, proportional to $\nabla s[16] ;$ here $e_{i}$ and $r_{i}$ are the flexo- and order-electric coefficients, respectively. Using the definition $\mathbf{N}=\sqrt{s} \hat{\mathbf{n}}$, and comparing the expressions above for $\mathbf{P}_{\mathrm{OF}}^{(\mathrm{ed})}$ and $\mathbf{P}_{\mathbf{N}}^{(\mathrm{ed})}$, Eq. (21), we obtain the following relations between the flexo- and order-electric coefficients and the electro-distortion coefficients $\xi_{1}(s)$ :

$$
\begin{aligned}
& e_{1}=s \xi_{1}+s^{2} \xi_{3}, \quad e_{3}=s \xi_{1}, \quad r_{1}=\xi_{1}+s\left(\xi_{3}+\xi_{4}\right), \\
& r_{2}=\xi_{2} .
\end{aligned}
$$

To compare $\mathbf{P}^{(\mathrm{ed})}$ in the nemator and LdG models we construct $\underline{\mathbf{P}}_{\mathbf{N}}^{(\text {ed) }}$ using the same diagrams, Fig. 4, and substituting $S_{i j} \rightarrow \underline{Q}_{i j}$, e.g., diagram $\mathbf{1 , 1}$ yields $\underline{\mathbf{p}}_{1,1}=\nabla \cdot \underline{\mathbf{Q}}$. We then

TABLE III. Numerical expansion coefficients $\left\langle\alpha, \beta \mid \alpha_{n}, \beta_{n}\right\rangle_{(\mathbf{p})}$ in Eq. (22), bold, and $\underline{\left\langle\alpha, \beta \mid \alpha_{n}, \beta_{n}\right\rangle}$ (p) in Eq. (24), underlined in

\begin{tabular}{|c|c|c|c|c|}
\hline & 1,1 & 1,2 & $2,1 \mathrm{a}$ & $2,2 \mathrm{a}$ \\
\hline 1,1 & $\mathbf{1}(\underline{1})$ & $(\underline{-1 / 3})$ & & \\
\hline 1,2 & & $\overline{1}$ & & \\
\hline $2,1 \mathrm{a}$ & $(-1 / 3)$ & $(\underline{1 / 9})$ & $\mathbf{1}(\underline{1})$ & $(-1 / 3)$ \\
\hline $2,1 \mathrm{~b}$ & $\overline{\mathbf{1}(2 / 3)}$ & $(\underline{1 / 9})$ & $-1(-1)$ & $\overline{1(2 / 3)}$ \\
\hline $2,2 \mathrm{a}$ & & & & $\overline{1}$ \\
\hline $2,2 \mathrm{~b}$ & & $\mathbf{1}(2 / 3)$ & & \\
\hline 3,1 & $(-2 / 9)$ & $(-\overline{1 / 27})$ & & $1(5 / 9)$ \\
\hline 3,2 & & $(\underline{-2 / 9)}$ & & $\mathbf{1}(\underline{2 / 3})$ \\
\hline
\end{tabular}
parentheses. expand $\underline{\mathbf{p}}_{\alpha, \beta}$ using the same basis

$$
\underline{\mathbf{p}}_{\alpha, \beta}=\sum_{n} \underline{\left\langle\alpha, \beta \mid \alpha_{n}, \beta_{n}\right\rangle}(\mathbf{p}) s^{\Delta \alpha} \mathbf{p}_{\alpha_{n}, \beta_{n}},
$$

where $\left\langle\alpha, \beta \mid \alpha_{n}, \beta_{n}\right\rangle_{(\mathbf{p})}$ are the numerical expansion coefficients shown in Table III.

Note that similar to the elastic terms $f_{\mathbf{N}}^{(\mathrm{el})}$ and $f_{\mathbf{N}}^{(\mathrm{el})}, \mathbf{P}_{\mathbf{N}}^{(\mathrm{ed})}$ and $\underline{\mathbf{P}}_{\mathbf{N}}^{(\text {ed })}$ are not equivalent either. When $\underline{\mathbf{P}}_{\mathbf{N}}^{(\text {ed })}$ is valid, then $\underline{\mathbf{p}}_{1,2} \equiv \underline{\mathbf{p}}_{2,2 a} \equiv 0$ and the electrodistortion coefficients $\xi_{1}(s)$ in Eq. (17) for $\mathbf{P}_{\mathbf{N}}^{(\text {ed) }}$ obey the relations

$$
\xi_{2}(0)=-\xi_{1}(0) / 3, \quad \xi_{4}(0)=\xi_{1}^{\prime}(0) .
$$

Equations (19), (20) and (25) are probably valid for the same materials and temperature ranges.

Surface anchoring and static equilibrium. Minimization of the total free energy $\Phi$, Eq. (1), is a multidimensional variational problem with soft boundary conditions, see, e.g., Ref. [42]. These conditions are controlled by the surface anchoring $w_{\mathbf{N}}\left(S_{i j}\right)$, which we present as

$$
w_{\mathbf{N}}=\frac{1}{2} w_{i j k l}\left(S_{i j}-S_{i j}^{(w)}\right)\left(S_{k l}-S_{k l}^{(w)}\right),
$$

where $S_{i j}^{(w)}=s^{(w)} n_{i}^{(w)} n_{j}^{(w)}$ is the optimal value for $S_{i j}$ tensor at the surface $\sigma$ that is defined by the easy axis $\hat{\mathbf{n}}^{(w)}$ and the optimal surface value $s^{(w)}$ for the order parameter $s$, and $w_{i j k l}$ are the elements of the positive-definite fourth order three dimensional strongly paired symmetric tensor $\mathbf{w}$, which is symmetric with respect to $i \leftrightarrow j, k \leftrightarrow l$, and $i j \leftrightarrow k l$ permutations and provides $w_{\mathbf{N}}\left(S_{i j}\right)>0$ for any $S_{i j}$. It is convenient to analyze the properties of $\mathbf{w}$ in the local orthogonal basis $\left\{\hat{\mathbf{e}}_{\mathbf{1}}, \hat{\mathbf{e}}_{2}, \hat{\mathbf{n}}^{(w)}\right\}$. If $\hat{\mathbf{n}}^{(w)}$ is not parallel to the outward normal unit vector $\hat{\boldsymbol{v}}$ to the surface $\sigma$ (surface alignment is not homeotropic), then $\hat{\mathbf{e}}_{\mathbf{1}}=\left(\hat{\boldsymbol{v}} \times \hat{\mathbf{n}}^{(w)}\right) /\left|\hat{\boldsymbol{v}} \times \hat{\mathbf{n}}^{(w)}\right|$ and $\hat{\mathbf{e}}_{2}=\hat{\mathbf{n}}^{(w)} \times \hat{\mathbf{e}}_{1}$; note that if $\hat{\mathbf{n}}^{(w)}$ is tangential to the surface, $\hat{\mathbf{n}}^{(w)} \cdot \hat{\boldsymbol{v}}=0$, then $\hat{\mathbf{e}}_{2}=\hat{\boldsymbol{v}}$. For the homeotropic alignment, $\hat{\mathbf{n}}^{(w)}=\hat{\boldsymbol{v}}, \hat{\mathbf{e}}_{1}$ and $\hat{\mathbf{e}}_{2}$ are tangential to the surface and can be chosen arbitrarily or based on the surface geometry. In the chosen basis, elements $w_{i j k l}$ have the following properties: (a) $w_{i j k l}=0$, if any index value appear an odd number of times; (b) $w_{1133}=w_{2233}=0$; (c) $w_{3333}$ is the anchoring coefficient for $\left(s-s^{(w)}\right)^{2}$ term, that penalizes deviation of the surface value of $s$ from $s^{(w)}$; (d) the elements $w_{1313}=4 s^{2} w_{a z}$ and $w_{2323}=4 s^{2} w_{\text {pol }}$ control the small deviations $\mathbf{N}$ from $\hat{\mathbf{n}}^{(w)}$ and are related, respectively, to the azimuthal $w_{a z}$ and polar $w_{\text {pol }}$ anchoring coefficients in the Rapini-Papoular anchoring potential $w_{R P}$ in the OF model

$$
w_{R P}=\frac{1}{2}\left[w_{a z}\left(\hat{\mathbf{n}} \cdot \hat{\mathbf{e}}_{\mathbf{1}}\right)^{2}+w_{\mathrm{pol}}\left(\hat{\mathbf{n}} \cdot \hat{\mathbf{e}}_{2}\right)^{2}\right] .
$$

Expression (26) is a generalization of the anchoring representation with a second rank tensor and covers various cases of surface anchoring in the LdG model [43]; e.g., it reproduces the isotropic anchoring around $\hat{\mathbf{n}}^{(w)}$ [44] with $w_{i j k l}=w_{0} \delta_{i k} \delta_{j l}$ and the degenerate tangential alignment [45-47] with $S_{i j}^{(w)}=$ $s^{(w)}\left(\delta_{i j}-v_{i} v_{j}\right)$.

The equilibrium state is determined by zero variation $\delta \Phi=0$ with respect to variations of the nemator field $\delta N_{i}$ and of the electric potential $\delta u$ in LC volume, when the electric field $\mathbf{E}=-\nabla u$ is applied; we consider fixed values of $u$ at the 
boundaries. Because $\Phi$ is a function of $S_{i j}$ rather than $N_{i}$, we apply the chain rule for variations $\delta S_{i j}=N_{i} \delta N_{j}+N_{j} \delta N_{i}$ and obtain

$$
\begin{aligned}
\delta \Phi= & 2 \int_{V} \frac{\delta f_{\mathbf{N}}}{\delta S_{i j}} N_{j} \delta N_{i} d V_{T}-\int_{V}\left(\frac{\partial f_{\mathbf{N}}}{\partial u_{, k}}\right)_{, k} \delta u d V \\
& +2 \int_{\sigma}\left(\frac{\partial f_{\mathbf{N}}}{\partial S_{i j, k}} \hat{v}_{k}+\frac{\partial w_{\mathbf{N}}}{\partial S_{i j}}\right) N_{j} \delta N_{i} d \sigma=0,
\end{aligned}
$$

where the variations in three integrals are independent and should vanish in equilibrium. The first integral requires zero static bulk nemator force field $\overline{\mathbf{h}}$

$$
\bar{h}_{i}=-\frac{\delta f_{\mathbf{N}}}{\delta N_{i}}=-2 N_{j}\left[\frac{\partial f_{\mathbf{N}}}{\partial S_{i j}}-\left(\frac{\partial f_{\mathbf{N}}}{\partial S_{i j, k}}\right)_{, k}\right]=0 .
$$

The second integral provides the equation for the electric displacement $\mathbf{D}=\varepsilon_{0} \boldsymbol{\varepsilon} \cdot \mathbf{E}+\mathbf{P}_{\mathbf{N}}^{(\mathrm{ed})}$ in LC with no free charges

$$
\nabla \cdot \mathbf{D}=0
$$

and the surface integral zeros the static surface nemator force field $\overline{\mathbf{h}}^{(\sigma)}$

$$
\bar{h}_{i}^{(\sigma)}=-2 N_{j}\left(\frac{\partial f_{\mathbf{N}}}{\partial S_{i j, k}} \hat{v}_{k}+\frac{\partial w_{\mathbf{N}}}{\partial S_{i j}}\right)=0 .
$$

Equations (29), (30), and (31) allow one to study and simulate the equilibrium structures with bulk and surface defects adequately, because when $\mathbf{N}$ loops around a semi-integer disclination, it flips at some point and $\overline{\mathbf{h}}$ and $\overline{\mathbf{h}}^{(\sigma)}$ flip with $\mathbf{N}$, but do not exhibit singularities.

\section{DYNAMIC EQUATIONS}

The dynamic equations for uniaxial NLC has been extensively studied using the director and tensor models, see e.g., Refs. [20-23,25,48,49] and references therein. Based on OF elasticity, Ericksen [20] and Leslie [21] employed the continuous mechanics approach in a uniaxial NLC and derived the dynamic equations for the hydrodynamic flow and director reorientation using the balance equations for mass, linear and angular momenta, energy, and entropy. The Harvard group has developed the hydrodynamic model, where only hydrodynamic (Goldstone) variables are considered [22,23]. Within the LdG approach the dynamic properties are usually described by the Beris-Edwards model [25] based on the Poisson bracket formalism.

In the absence of the electric and magnetic fields the free energy of uniform uniaxial NLC depends on the nemator amplitude in $f_{\mathbf{N}}^{\text {(micro) }}(s)$, but does not on its direction because $f_{\mathbf{N}}^{\text {(macro) }}\left(S_{i j}, S_{k l, m}\right)=0$. Thus, the nemator direction is a hydrodynamic variable and its amplitude is not. We, therefore, develop the nemator dynamic model using the continuous mechanics approach, similar to the Ericksen-Leslie model, but with two modifications. First, the nemator $\mathbf{N}$ has a variable length and therefore is entirely determined by the dynamic equations. Second, the resulting dynamic equations should depend on $S_{i j, k}$ rather than on $N_{i, k}$ to allow $\mathbf{N} \leftrightarrow-\mathbf{N}$ flip, required for description of a system with semi-integer disclinations. We derive the dynamic equations for the nemator $\mathbf{N}(\mathbf{r}, t)$ and flow velocity $\mathbf{v}(\mathbf{r}, t)$ using the conservation laws of mass, linear and angular momenta, and energy for a NLC fluid parcel of the volume $V_{p}$ bounded by the surface $\sigma_{p}$. For simplicity we consider a uniaxial NLC to be incompressible and neglect effects of diffusion, temperature change, and ionic movement.

(a) The incompressibility condition, $\rho=$ const results in

$$
\operatorname{div} \mathbf{v}=v_{i, i}=0 .
$$

(b) The linear momentum balance is

$$
\frac{d}{d t} \int_{V_{p}} \rho \mathbf{v} d V=\int_{V_{p}} \rho \mathbf{F} d V+\int_{\sigma_{p}} \mathbf{T} \cdot \hat{\mathbf{v}}_{p} d \sigma,
$$

where $\mathbf{F}$ is the body force per unit mass, $\mathbf{T}$ is the stress tensor, $\hat{\mathbf{v}}_{p}$ is the outward normal unit vector to the surface $\sigma_{p}$, and $\frac{d}{d t}$ is the material time derivative also denoted below by the superposed dot. The arbitrary choice of $V_{p}$ allows one to obtain the linear momentum balance in differential form

$$
\rho \dot{v}_{i}=\rho F_{i}+T_{i j, j},
$$

where $\dot{v}_{i}=d v_{i} / d t=v_{i}^{\prime}+v_{i, j} v_{j}$.

(c) The angular momentum balance is

$$
\begin{aligned}
& \frac{d}{d t} \int_{V_{p}} \rho(\mathbf{r} \times \mathbf{v}+I \mathbf{N} \times \dot{\mathbf{N}}) d V \\
& =\int_{V_{p}} \rho(\mathbf{r} \times \mathbf{F}+\mathbf{N} \times \mathbf{g}) d V \\
& \quad+\int_{\sigma_{p}}\left(\mathbf{r} \times \mathbf{T} \cdot \hat{\boldsymbol{v}}_{p}+\mathbf{N} \times \mathbf{H} \cdot \hat{\boldsymbol{v}}_{p}\right) d \sigma,
\end{aligned}
$$

where $I$ is the moment of inertia per unit mass, $\mathbf{g}$ is the external nemator force per unit mass, and $\mathbf{H}$ is the nemator stress tensor. Following Leslie [50], we introduce the intrinsic force density $\mathbf{h}$, which in our case includes the contribution of $\mathbf{H}$ and controls the nemator dynamics

$$
\rho I \ddot{N}_{i}=h_{i}+\rho g_{i} .
$$

Using Eqs. (34) and (36), we rewrite the angular momentum balance (35) in differential form as

$$
\varepsilon_{i j k}\left[N_{j} h_{k}-T_{k j}-\left(N_{j} H_{k l}\right)_{, l}\right]=0 .
$$

(d) In the energy balance, the viscous losses defined by the dissipation function $R$ and changes of the kinetic energy, in parentheses, and the nematic free energy $f$ are powered by body and surface forces, right side of the equation,

$$
\begin{aligned}
& \frac{d}{d t} \int_{V_{p}}\left[\frac{1}{2} \rho(\mathbf{v} \cdot \mathbf{v}+I \dot{\mathbf{N}} \cdot \dot{\mathbf{N}})+f_{\mathbf{N}}\right] d V+\int_{V_{p}} R d V \\
& \quad=\int_{V_{p}} \rho(\mathbf{v} \cdot \mathbf{F}+\dot{\mathbf{N}} \cdot \mathbf{g}) d V+\int_{\sigma_{p}}\left(\mathbf{v} \cdot \mathbf{T} \cdot \hat{\mathbf{v}}_{p}+\dot{\mathbf{N}} \cdot \mathbf{H} \cdot \hat{\mathbf{v}}_{p}\right) d \sigma .
\end{aligned}
$$

Using the chain rule for partial derivatives and noncommutativity of the material time derivative and the gradient $\frac{d}{d t}\left(S_{i j, k}\right)=\left(\dot{S}_{i j}\right)_{, k}-S_{i j, l} v_{l, k}$, we derive $\dot{f}_{\mathbf{N}}$ as

$$
\begin{aligned}
\dot{f}_{\mathbf{N}}= & 2 \dot{N}_{i}\left(\frac{\partial f_{\mathbf{N}}}{\partial S_{i j}} N_{j}+\frac{\partial f_{\mathbf{N}}}{\partial S_{i j, k}} N_{j, k}\right)+\frac{\partial f_{\mathbf{N}}}{\partial S_{k l, j}} S_{k l, i} v_{i, j} \\
& -2 \frac{\partial f_{\mathbf{N}}}{\partial S_{i k, j}} N_{k}\left(\dot{N}_{i}\right)_{, j} .
\end{aligned}
$$


Substituting Eqs. (34), (36), and (39) in Eq. (38) we obtain

$$
\begin{aligned}
R= & v_{i, j}\left(T_{i j}+\frac{\partial f_{\mathbf{N}}}{\partial S_{k l, j}} S_{k l, i}\right)+\left(\dot{N}_{i}\right)_{, j}\left(H_{i j}-2 \frac{\partial f_{\mathbf{N}}}{\partial S_{i k, j}} N_{k}\right) \\
& -\dot{N}_{i}\left(h_{i}-H_{i j, j}+2 \frac{\partial f_{\mathbf{N}}}{\partial S_{i j}} N_{j}+2 \frac{\partial f_{\mathbf{N}}}{\partial S_{i j, k}} N_{j, k}\right) .
\end{aligned}
$$

The non-negativity of the dissipation function $R \geqslant 0$ for any dynamic process leads to the following expressions:

$$
\begin{gathered}
T_{i j}=-\frac{\partial f_{\mathbf{N}}}{\partial S_{k l, j}} S_{k l, i}-p \delta_{i j}+\tilde{T}_{i j}, \\
H_{i j}=2 \frac{\partial f_{\mathbf{N}}}{\partial S_{i k, j}} N_{k}+\tilde{H}_{i j}, \\
h_{i}=H_{i j, j}-2 \frac{\partial f_{\mathbf{N}}}{\partial S_{i j}} N_{j}-2 \frac{\partial f}{\partial S_{i j, k}} N_{j, k}-\tilde{h}_{i},
\end{gathered}
$$

where $p$ is the pressure, determined from the incompressibility condition (32) and $\tilde{T}_{i j}, \tilde{h}_{i}$, and $\tilde{H}_{i j}$ are the viscous parts of the corresponding tensors and vector. We select the negative sign for $\tilde{h}_{i}$ to make all terms in Eq. (40) positive; thus $\tilde{h}_{i}$ is the thermodynamic flux. Following the Leslie approach [50], we assume that $R$ does not depend on nonobjective $\left(\dot{N}_{i}\right)_{, j}$; thus $\tilde{H}_{i j}=0$ and Eq. (40) reduces to $R=v_{i, j} \tilde{T}_{i j}+\dot{N}_{i} \tilde{h}_{i}$. Combining Eqs. (42) and (43) we obtain

$$
h_{i}=-2\left[\frac{\partial f_{\mathbf{N}}}{\partial S_{i j}}-\left(\frac{\partial f_{\mathbf{N}}}{\partial S_{i j, k}}\right)_{, k}\right] N_{j}-\tilde{h}_{i}=\bar{h}_{i}-\tilde{h}_{i} .
$$

Note that the static force $\overline{\mathbf{h}}$ is a reactive part of $\mathbf{h}$.

We split the velocity gradient $v_{i, j}=A_{i j}+W_{i j}$ into the symmetric rate of strain tensor $A_{i j}=\frac{1}{2}\left(v_{i, j}+v_{i, j}\right)$ and the skew-symmetric vorticity tensor $W_{i j}=\frac{1}{2}\left(v_{i, j}-v_{j, i}\right)$ and the viscous stress tensor $\tilde{T}_{i j}=\tilde{T}_{i j}^{s}+\tilde{T}_{i j}^{a}$ into the symmetric $\tilde{T}_{i j}^{s}$ and the skew-symmetric $\tilde{T}_{i j}^{a}$ parts. The latter is determined from the angular momentum balance (37)

$$
\tilde{T}_{i j}^{a}=\frac{1}{2}\left(\tilde{h}_{j} N_{i}-\tilde{h}_{i} N_{j}\right),
$$

and allows us to express $R$ as a sum of products of the objective (frame-indifferent) thermodynamic forces $A_{i j}$ and $\stackrel{\circ}{N_{i}}=$ $\dot{N}_{i}-W_{i j} N_{j}$ with the corresponding thermodynamic fluxes $\tilde{T}_{i j}^{s}$ and $\tilde{h}_{i}$

$$
R=A_{i j} \tilde{T}_{i j}^{s}+\tilde{h}_{i} \stackrel{\circ}{N}_{i}
$$

Because of Eq. (45), $\tilde{T}_{i j}$ is also objective and the constitutive equations can be written for $\tilde{T}_{i j}$ and $\tilde{h}_{i}$ as linear functions of the thermodynamic forces $A_{i j}$ and $\stackrel{\circ}{N_{i}}$ :

$$
\begin{gathered}
\tilde{T}_{i j}=\alpha_{1} N_{i} N_{j} N_{k} A_{k l} N_{l}+\alpha_{2} \stackrel{\circ}{N_{i}} N_{j}+\alpha_{3} N_{i} \stackrel{\circ}{N_{j}}+\alpha_{4} A_{i j} \\
+\alpha_{5} N_{j} A_{i k} N_{k}+\alpha_{6} N_{i} A_{j k} N_{k}+\alpha_{7} N_{i} N_{j} N_{k} \stackrel{\circ}{N_{k}}, \\
\tilde{h}_{i}=\gamma_{1} \stackrel{\circ}{N_{i}}+\gamma_{2} A_{i j} N_{j}+\gamma_{3} N_{i} N_{j} \stackrel{\circ}{N_{j}}+\gamma_{4} N_{i} N_{j} A_{j k} N_{k},
\end{gathered}
$$

where $\alpha_{l}$ and $\gamma_{m}$ are the phenomenological viscous coefficients, which are functions of $s=\operatorname{Tr} \mathbf{S}=\mathbf{N} \cdot \mathbf{N}$ and obey the relations $\gamma_{1}=\alpha_{3}-\alpha_{2}, \gamma_{2}=\alpha_{6}-\alpha_{5}$ because of Eq. (45), and $\gamma_{2}=\alpha_{3}+\alpha_{2}, \gamma_{4}=\alpha_{7}$ from the Onsager reciprocal relations.

Comparison of Eq. (48) with the corresponding expression in the EL model provides the relations between $\alpha_{1-6}$ and $\gamma_{1,2}$ and the Leslie coefficients $\alpha_{1-6}^{(L)}$ and $\gamma_{1,2}^{(L)}$

$$
\begin{gathered}
\alpha_{4}^{(L)}=\alpha_{4}, \quad \alpha_{1}^{(L)}=s^{2} \alpha_{1}, \quad \alpha_{l}^{(L)}=s \alpha_{l}, \\
l=2,3,5,6, \quad \text { and } \quad \gamma_{1,2}^{(L)}=s \gamma_{1,2} .
\end{gathered}
$$

The remaining $\alpha_{7}$ and $\gamma_{3,4}$ describe the viscosities associated with the change of order parameter $\mathrm{s}$ and can be expressed through the coefficients $\beta_{1-3}$ introduced by Ericksen [7]

$$
\beta_{1}=s \alpha_{7} / 2, \quad \beta_{2}=\gamma_{3} / 4, \quad \beta_{3}=s \gamma_{4} / 2 .
$$

We assume that all $\alpha_{l}$ and $\gamma_{m}$ in constitutive Eqs. (47) and (48) have finite nonzero limits when $s \rightarrow 0$. Calculations of the Leslie coefficients $\alpha_{l}^{(L)}$ in Marrucci [51], Kuzuu-Doi [52], and Osipov-Terentjev [53] models support this assumption; the only difference is that $\alpha_{1}^{(L)} \propto\left\langle P_{4}\right\rangle$ in the Osipov-Terentjev model instead of $\alpha_{1}^{(L)} \propto s^{2}$ in Eq. (49). Thus in the first approximation, $\alpha_{l}$ and $\gamma_{m}$ can be considered constant for modeling dynamic processes in systems with inhomogeneous orientational order and defects.

(e) For surface dynamic equations, when NLC is in a cell or capillary, the bulk dynamic Eqs. (32), (34), and (36) for $\mathbf{v}(\mathbf{r}, t), \mathbf{N}(\mathbf{r}, t)$ and $p(\mathbf{r}, t)$ should be supplemented with the surface conditions. We consider the standard geometry of solid surfaces, which are fixed, sliding or rotating, like in viscosity measurements. For the nonslipping condition $\mathbf{v}(\mathbf{r}, t)$ at the surfaces coincides with the velocity of the solid substrate. The nemator dynamic equation at the surface is simpler than the bulk one, Eq. (36),

$$
h_{i}^{(\sigma)}=\bar{h}_{i}^{(\sigma)}-\tilde{h}_{i}^{(\sigma)}=0,
$$

because the inertial term and external forces are negligibly small. We also neglect the effects of nonlocality in surface interactions investigated by Durand and Virga [54] and adopt the viscous force $\tilde{\mathbf{h}}^{(\sigma)}$ similar to $\tilde{\mathbf{h}}$ (48), but without the terms associated with gradients of the velocity at the surface

$$
\tilde{h}_{i}^{(\sigma)}=\gamma_{1}^{(\sigma)} \dot{N}_{i}+\gamma_{3}^{(\sigma)} N_{i} N_{j} \dot{N}_{j},
$$

where $\gamma_{m}^{(\sigma)}$ are the surface viscous coefficients. The surface viscosities $\gamma_{m}^{(\sigma)}$ are small, as the experimental data reveals that $\gamma_{1}^{(\sigma)} / \gamma_{1} \sim 0.01-1 \mu \mathrm{m}$ [55-57] and the second term with $\gamma_{3}^{(\sigma)}$, which $\propto \dot{s}$ at the surface, is probably even smaller. Thus, the static Eq. (31), $\overline{\mathbf{h}}^{(\sigma)}=0$, may be also used as the nemator boundary condition for the dynamic processes.

The slow surface processes, such as sliding and gliding, are caused by modification of soft polymer surfaces [58-62] or by adsorption of dopants from a liquid crystal [63]; they can be modeled through the additional dynamic equations for the parameters in the surface anchoring $w_{\mathbf{N}}$, Eq. (26).

\section{DISCUSSION}

The proposed nemator model is not the first attempt to describe NLCs with a vector of the variable length that 
incorporates information of the direction and the degree of molecular arrangement. Ericksen discussed and rejected the possibility of using the vector $\mathbf{m}=\operatorname{sgn}(s) \sqrt{|s|} \hat{\mathbf{n}}$ because the energy terms have different expressions for positive and negative $s$ [7]. Lin introduced the vector $\mathbf{u}=s \hat{\mathbf{n}}$ to study the existence of minimizers for the single-elastic-constant version of the nematic free energy [64]. Calderer et al. employed the single-elastic-constant model with the vector $\mathbf{u}$ to investigate properties of NLC composites with ferromagnetic inclusions [65]. To improve numerical schemes for modeling flows in NLCs $\hat{\mathbf{n}}$ was substituted with the variable length vector $\mathbf{d}$, and the rigid condition $|\hat{\mathbf{n}}|=1$ was replaced with the penalty energy term $F(\mathbf{d})=\left(|\mathbf{d}|^{2}-1\right)^{2} / 4 \varepsilon^{2}[66,67]$.

There are two drawbacks of the nemator model, which ought to be addressed in the future. First, the presented version of the model is not capable to describe a spontaneous biaxial phase as well as the field-induced biaxiality [68] and local biaxiality near semi-integer disclinations [17-19] in a uniaxial NLC. Second, the nemator model seems inapplicable for a uniaxial NLC with negative $s$, because $\mathbf{N}$ is imaginary. However, the static Eqs. (29), (31), and dynamic Eqs. (36), (51) equations for $\mathbf{N}(\mathbf{r}, t)$ look valid because the external force $\mathbf{g}$ and the intrinsic forces $\mathbf{h}$, Eqs. (44), (48), and $\mathbf{h}^{(\sigma)}$, and Eqs. (31) and (52), also have imaginary values. The further study should investigate the nemator model validity for the systems with $s<0$ and with mixed, $s>0$ and $s<0$, regions.

In conclusion, the nemator model provides an alternative way to analyze and simulate the static and dynamic properties of uniaxial NLCs with various patterns and defects. The static patterns can be reconstructed as an equilibrium nemator field $\mathbf{N}(\mathbf{r}, t)$ determined by direct minimization of the total free energy $\Phi$, Eq. (1), or by solving Eqs. (29) and (31) for bulk $\overline{\mathbf{h}}$ and surface $\overline{\mathbf{h}}^{(\sigma)}$ nemator force fields, respectively. To study the dynamic properties, the nemator model provides the complete set of dynamic Eqs. (32), (34), and (36) for $\mathbf{v}(\mathbf{r}, t)$, $\mathbf{N}(\mathbf{r}, t)$ and $p(\mathbf{r}, t)$, where the stress tensor $\mathbf{T}$ is defined by Eqs. (41) and (47), and the intrinsic nemator force density $\mathbf{h}$ is defined by Eqs. (44) and (48). For simplicity, the equations are derived under conditions of constant density and temperature, but the generalization is rather straightforward. The model is suitable for a description of a nematic with point and linear defects, because the dynamic equations do not contain spatial derivatives of $\mathbf{N}(\mathbf{r}, t)$ and are either symmetric, Eqs. (32) and (34), or antisymmetric, Eq. (36), with respect to the local flip $\mathbf{N} \leftrightarrow-\mathbf{N}$. In the usually applied noninertial limit, $I=0$ in Eq. (36), the equations contain only first time derivatives of $\mathbf{v}(\mathbf{r}, t)$ and $\mathbf{N}(\mathbf{r}, t)$, and the numerical modeling simplifies. Within this model, the interaction of $\mathbf{N}$ with applied electric and magnetic fields can be described either by the intrinsic nemator force density $\mathbf{h}$ via including interaction in $f_{\mathbf{N}}$ or by the external nemator force $\mathbf{g}$. The former description is probably more convenient for dc and stationary high frequency ac fields, whereas the latter is required for nonstationary conditions.
The proposed nemator model is equivalent to the director and Q-tensor models in their validity domains for uniaxial NLCs. This equivalency justifies applicability of the nemator model in the entire uniaxial nematic phase and allows one to determine the nemator model material parameters from the existing experimental data.

In our opinion, the proposed nemator representation and model possess certain advantages over the existing director and Q-tensor representations and models. Comparison of the director $\hat{\mathbf{n}}$ and nemator $\mathbf{N}=\sqrt{s} \hat{\mathbf{n}}$ representations reveals that the latter is more efficient because it contains the additional information on the uniaxial orientational order $s$ and identifies linear and points defect cores in uniaxial NLCs from the condition $\mathbf{N}=0$, whereas $\hat{\mathbf{n}}$ is undefined in the defect cores. The equations in the nemator model contain spatial derivatives of the dyadic tensor $\mathbf{S}=\mathbf{N} \otimes \mathbf{N}$ rather than derivatives of $\mathbf{N}$, and thus are insensitive to the flip, $\mathbf{N} \rightarrow-\mathbf{N}$ in any point of space. This feature allows one to simulate semi-integer disclinations and provides a broader range of applicability for the nemator model than for the OF, gOF, and EL models. Currently, semiinteger disclinations are simulated in Q-tensor representation using the LdG and Beris-Edwards models; however, $\mathbf{N}$ (three variables) provides a more efficient representation than $\mathbf{Q}$ (five variables) and the dyadic nature of the $\mathbf{S}$ tensor allows one to avoid a polynomial expansion and to build the free energy and dynamic equations in the complete form.

The nemator model can also serve as a starting point to build a model for active uniaxial soft matter systems, because it (a) uses the vector $\mathbf{N}(\mathbf{r}, t)$, which defines the amplitude and direction of orientational ordering in a uniaxial nonpolar system and has limited length $|\mathbf{N}(\mathbf{r}, t)| \leqslant 1$ controlled by the proposed microscopic part of free energy $f_{\mathbf{N}}^{\text {(micro) }}$, Eq. (5), where $T^{*}$ and the temperature dependences of coefficients $a^{\prime}$, $b^{\prime}$, and $c^{\prime}$ can be chosen according to specifics of an active system; (b) is based on the continuous mechanics approach and balance equations that allows one to incorporate naturally the required active forces and energy terms; (c) provides the advanced representation, Eq. (26), of the surface anchoring; (d) contains the material parameters, which are constant in the first approximation and, therefore, convenient for modeling; and (e) describes generation and recombination of defects.

\section{ACKNOWLEDGMENTS}

This research was supported by the National Science Foundation (NSF) under Award No. DMS-1729509 (derivation of bulk free energy and dynamic equations) and the U.S. Department of Energy (DOE), Office of Science, Basic Energy Sciences (BES), under Award No. DE-SC0019105 (analysis of surface anchoring and surface equations). The work of N.A. was partially supported by the National Academy of Sciences of Ukraine within the projects BC No. 202 and BC No. 197. Discussions with O. D. Lavrentovich, M. C. Calderer, D. Golovaty, and N. J. Walkington are appreciated.
[1] T. Sluckin, D. Dunmur, and H. Stegemeyer, Crystals that Flow: Classic Papers from the History of Liquid Crystals (Taylor \& Francis, New York, 2004).
[2] M. C. Marchetti, J. F. Joanny, S. Ramaswamy, T. B. Liverpool, J. Prost, M. Rao, and R. A. Simha, Hydrodynamics of soft active matter, Rev. Mod. Phys. 85, 1143 (2013). 
[3] F. Julicher, S. W. Grill, and G. Salbreux, Hydrodynamic theory of active matter, Rep. Prog. Phys. 81, 076601 (2018).

[4] C. W. Oseen, The theory of liquid crystals, Trans. Faraday Society 29, 883 (1933).

[5] F. C. Frank, I. Liquid crystals. On the theory of liquid crystals, Discuss. Faraday Soc. 25, 19 (1958).

[6] A. Poniewierski and T. J. Sluckin, On the free-energy density of non-uniform nematics, Mol. Phys. 55, 1113 (1985).

[7] J. L. Ericksen, Liquid crystals with variable degree of orientation, Arch. Ration. Mech. Anal. 113, 97 (1991).

[8] J. M. Ball and A. Zarnescu, Orientable and non-orientable line field models for uniaxial nematic liquid crystals, Mol. Cryst. Liq. Cryst. 495, 221 (2008).

[9] J. M. Ball and A. Majumdar, Nematic liquid crystals: From Maier-Saupe to a continuum theory, Mol. Cryst. Liq. Cryst. 525, 1 (2010).

[10] J. M. Ball and S. J. Bedford, Discontinuous order parameters in liquid crystal theories, Mol. Cryst. Liq. Cryst. 612, 1 (2015).

[11] P.-G. d. Gennes and J. Prost, The Physics of Liquid Crystals (Clarendon Press, Oxford, 1993).

[12] M. Kleman and O. D. Lavrentovich, Soft Matter Physics: An Introduction (Springer, New York, 2003)

[13] L. Longa, D. Monselesan, and H. R. Trebin, An extension of the Landau-Ginzburg-Degennes theory for liquid-crystals, Liq Cryst 2, 769 (1987)

[14] L. Longa and H. R. Trebin, Structure of the elastic freeenergy for chiral nematic liquid-crystals, Phys. Rev. A 39, 2160 (1989).

[15] R. B. Meyer, Piezoelectric Effects in Liquid Crystals, Phys. Rev. Lett. 22, 918 (1969).

[16] G. Barbero, I. Dozov, J. F. Palierne, and G. Durand, Order Electricity and Surface Orientation in Nematic Liquid-Crystals, Phys. Rev. Lett. 56, 2056 (1986).

[17] I. F. Lyuksyutov, Topological instability of singularities at small distances in nematics, Zh. Eksp. Teor. Fiz. 75, 358 (1978).

[18] N. Schopohl and T. J. Sluckin, Defect Core Structure in Nematic Liquid-Crystals, Phys. Rev. Lett. 59, 2582 (1987).

[19] S. Zhou, S. V. Shiyanovskii, H. S. Park, and O. D. Lavrentovich, Fine structure of the topological defect cores studied for disclinations in lyotropic chromonic liquid crystals, Nat. Commun. 8, 14974 (2017)

[20] J. L. Ericksen, Conservation laws for liquid crystals, Trans. Soc. Rheol. 5, 23 (1961).

[21] F. M. Leslie, Some constitutive equations for liquid crystals, Arch. Ration. Mech. Anal. 28, 265 (1968).

[22] D. Forster, T. C. Lubensky, P. C. Martin, J. Swift, and P. S. Pershan, Hydrodynamics of Liquid Crystals, Phys. Rev. Lett. 26, 1016 (1971).

[23] P. C. Martin, O. Parodi, and P. S. Pershan, Unified hydrodynamic theory for crystals, liquid crystals, and normal fluids, Phys. Rev. A 6, 2401 (1972).

[24] S. Hess, Irreversible thermodynamics of nonequilibrium alignment phenomena in molecular liquids and in liquid-crystals .1. derivation of nonlinear constitutive laws, relaxation of alignment, phase-transition, Z. Naturforsch. A 30, 728 (1975).

[25] A. N. Beris and B. J. Edwards, Thermodynamics of Flowing Systems: With Internal Microstructure (Oxford University Press, Oxford, 1994), Chap. 11.

[26] A. M. Sonnet and E. G. Virga, Dynamics of dissipative ordered fluids, Phys. Rev. E 64, 031705 (2001).
[27] G. Toth, C. Denniston, and J. M. Yeomans, Hydrodynamics of Topological Defects in Nematic Liquid Crystals, Phys. Rev. Lett. 88, 105504 (2002).

[28] W. Maier and A. Saupe, Eine einfache molekular-statistische theorie der nematischen kristallinflussigen phase 2, Z. Naturforsch. A 15, 287 (1960).

[29] V. I. Sugakov and S. V. Shiyanovskii, Phase transitions in confined liquid crystals, Ukr. Fiz. Zh. 22, 1441 (1977).

[30] J. Katriel, G. F. Kventsel, G. R. Luckhurst, and T. J. Sluckin, Free-energies in the Landau and molecular-field approaches, Liq Cryst 1, 337 (1986).

[31] Incomplete Beta Functions, in NIST Digital Library of Mathematical Functions, edited by F. W. J. Olver, A. B. O. Daalhuis, D. W. Lozier, B. I. Schneider, R. F. Boisvert, C. W. Clark, B. R. Miller, B. V. Saunders, H. S. Cohl, and M. A. McClain, http://dlmf.nist.gov/.

[32] G. R. Luckhurst, S. Naemura, T. J. Sluckin, K. S. Thomas, and S. S. Turzi, Molecular-field-theory approach to the Landau theory of liquid crystals: Uniaxial and biaxial nematics, Phys. Rev. E 85, 031705 (2012)

[33] S. V. Shiyanovskii, Free-energy power expansion for orientationally ordered phases: Energy and entropy, Phys. Rev. E 87, 060502(R) (2013).

[34] N. V. Madhusudana and R. Pratibha, Elasticity and orientational order in some cyanobiphenyls: Part IV reanalysis of the data, Mol. Cryst. Liq. Cryst. 89, 249 (1982).

[35] H. J. Coles, Laser and electric-field induced birefringence studies on cyanobiphenyl homologs, Mol. Cryst. Liq. Cryst. 49, 67 (1978).

[36] G. Barbero and C. Oldano, Derivative-dependent surfaceenergy terms in nematic liquid-crystals, Nuovo Cimento D 6 , 479 (1985).

[37] C. Oldano and G. Barbero, Possible boundary discontinuities of the tile angle in nematic liquid-crystals, J. Phys. Lett.-Paris $\mathbf{4 6}$ 451 (1985).

[38] Y. A. Nastishin, H. Liu, S. V. Shiyanovskii, O. D. Lavrentovich, A. F. Kostko, and M. A. Anisimov, Pretransitional fluctuations in the isotropic phase of a lyotropic chromonic liquid crystal, Phys. Rev. E 70, 051706 (2004).

[39] Y. A. Nastishin, H. Liu, T. Schneider, V. Nazarenko, R. Vasyuta, S. V. Shiyanovskii, and O. D. Lavrentovich, Optical characterization of the nematic lyotropic chromonic liquid crystals: Light absorption, birefringence, and scalar order parameter, Phys. Rev. E 72, 041711 (2005).

[40] T. Ostapenko, Y. A. Nastishin, P. J. Collings, S. N. Sprunt, O. D. Lavrentovich, and J. T. Gleeson, Aggregation, pretransitional behavior, and optical properties in the isotropic phase of lyotropic chromonic liquid crystals studied in high magnetic fields, Soft Matter 9, 9487 (2013).

[41] D. M. Agra-Kooijman, G. Singh, A. Lorenz, P. J. Collings, H S. Kitzerow, and S. Kumar, Columnar molecular aggregation in the aqueous solutions of disodium cromoglycate, Phys. Rev. E 89, 062504 (2014).

[42] I. M. Gelfand and S. V. Fomin, Calculus of Variations (PrenticeHall, Englewood Cliffs, NJ, 1963).

[43] M. Ravnik and S. Zumer, Landau-de Gennes modelling of nematic liquid crystal colloids, Liq. Cryst. 36, 1201 (2009).

[44] M. Nobili and G. Durand, Disorientation-induced disordering at a nematic-liquid-crystal solid interface, Phys. Rev. A 46, R6174 (1992). 
[45] T. J. Sluckin and A. Poniewierski, Novel Surface Phase-Transition in Nematic Liquid-Crystals-Wetting and the Kosterlitz-Thouless Transition, Phys. Rev. Lett. 55, 2907 (1985).

[46] A. K. Sen and D. E. Sullivan, Landau-de Gennes theory of wetting and orientational transitions at a nematic-liquid-substrate interface, Phys. Rev. A 35, 1391 (1987).

[47] J. B. Fournier and P. Galatola, Modeling planar degenerate wetting and anchoring in nematic liquid crystals, Europhys. Lett. 72, 403 (2005).

[48] A. Kilian and S. Hess, Derivation and application of an algorithm for the numerical-calculation of the local orientation of nematic liquid-crystals, Z. Naturforsch. A 44, 693 (1989).

[49] A. Sonnet, A. Kilian, and S. Hess, Alignment tensor versus director - description of defects in nematic liquid-crystals, Phys Rev E 52, 718 (1995).

[50] F. M. Leslie, Theory of flow phenomena in liquid crystals, in Advances in Liquid Crystals, edited by G. H. Brown (Elsevier, Amsterdam, 1979), Vol. 4, p. 1.

[51] G. Marrucci, Prediction of leslie coefficients for rodlike polymer nematics, Mol. Cryst. Liq. Cryst. 72, 153 (1982).

[52] N. Kuzuu and M. Doi, Constitutive equation for nematic liquidcrystals under weak velocity-gradient derived from a molecular kinetic-equation. II. leslie coefficients for rodlike polymers, J. Phys. Soc. Jpn. 53, 1031 (1984).

[53] M. A. Osipov and E. M. Terentjev, Rotational diffusion and rheological properties of liquid-crystals, Z. Naturforsch. A 44, 785 (1989).

[54] G. E. Durand and E. G. Virga, Hydrodynamic model for surface nematic viscosity, Phys. Rev. E 59, 4137 (1999).

[55] A. Mertelj and M. Copic, Surface-Dominated Orientational Dynamics and Surface Viscosity in Confined Liquid Crystals, Phys. Rev. Lett. 81, 5844 (1998).

[56] M. Vilfan, I. D. Olenik, A. Mertelj, and M. Copic, Aging of surface anchoring and surface viscosity of a nematic liquid crystal on photoaligning poly-(vinyl-cinnamate), Phys. Rev. E 63, 061709 (2001).
[57] P. Oswald, Measurement with a rotating magnetic field of the surface viscosity of a nematic liquid crystal, Europhys. Lett. 100, 26001 (2012).

[58] P. Vetter, Y. Ohmura, and T. Uchida, Study of memory alignment of nematic liquid-crystals on polyvinyl-alcohol coatings, Jpn. J. Appl. Phys. 32, L1239 (1993).

[59] V. P. Vorflusev, H. S. Kitzerow, and V. G. Chigrinov, Azimuthal surface gliding of a nematic liquid crystal, Appl. Phys. Lett. 70, 3359 (1997).

[60] I. Janossy and T. I. Kosa, Gliding of liquid crystals on soft polymer surfaces, Phys. Rev. E 70, 052701 (2004).

[61] S. Joly, K. Antonova, P. Martinot-Lagarde, and I. Dozov, Zenithal gliding of the easy axis of a nematic liquid crystal, Phys. Rev. E 70, 050701(R) (2004).

[62] N. Aryasova and Y. Reznikov, Liquid crystal alignment at macroscopically isotropic polymer surfaces: Effect of an isotropic-nematic phase transition, Phys. Rev. E 94, 032702 (2016).

[63] E. Ouskova, Y. Reznikov, S. V. Shiyanovskii, L. Su, J. L. West, O. V. Kuksenok, O. Francescangeli, and F. Simoni, Photoorientation of liquid crystals due to light-induced desorption and adsorption of dye molecules on an aligning surface, Phys. Rev. E 64, 051709 (2001).

[64] F. H. Lin, On nematic liquid-crystals with variable degree of orientation, Commun. Pure Appl. Math. 44, 453 (1991).

[65] M. C. Calderer, A. Desimone, D. Golovaty, and A. Panchenko, An effective model for nematic liquid crystal composites with ferromagnetic inclusions, Siam J. Appl. Math. 74, 237 (2014).

[66] F. H. Lin and C. Liu, Nonparabolic dissipative systems modeling the flow of liquid-crystals, Commun. Pure Appl. Math. 48, 501 (1995).

[67] C. Liu and N. J. Walkington, Mixed methods for the approximation of liquid crystal flows, Esaim-Math Model Num 36, 205 (2002).

[68] V. Borshch, S. V. Shiyanovskii, B. X. Li, and O. D. Lavrentovich, Nanosecond electro-optics of a nematic liquid crystal with negative dielectric anisotropy, Phys. Rev. E 90, 062504 (2014) 